\title{
Efficient Approach for Simulating Aperiodic Flows Due to Geometry Distortions
}

\author{
Feng Wang * and Luca di Mare ${ }^{\dagger}$ \\ Department of Engineering Science, University of Oxford
}

This paper presents an efficient approach for modelling aperiodic flows in an assembly due to geometry distortions. Firstly, a relation is established in physical space between grid displacement and flow perturbation based on linear theory. This relation is then extended to an assembly of components and projected to the Fourier space. A relation is thus constructed between grid displacement and flow perturbation in the frequency domain. Finally, the nonlinear coupling of the passage-averaged mean flow and the flow variations in the assembly is introduced. The capability of the proposed method is demonstrated on the outlet guide vane of a gas turbine engine with harmonic geometry distortions. Good agreement between the proposed method and the whole assembly computation is observed for the predicted flow perturbations in the assembly. This paper also demonstrates that the harmonic flow perturbation due to each harmonic geometry distortion can be used as a set of basis solutions to generate flow fields related to new combinations of geometry distortions. This has the potential to significantly reduce the computational cost when onerous tasks are expected for an iterative design processes of assemblies with geometry distortions.

\section{Nomenclature}

$$
\begin{aligned}
A & =\text { Roe matrix } \\
c & =\text { axial chord length } \\
e & =\text { internal energy } \\
E & =\text { total internal energy } \\
F & =\text { physical flux } \\
G \quad & =\text { right hand side for the linearized flow with grid displacement } \\
h & =\text { enthalpy } \\
H \quad & =\text { total enthalpy } \\
i & =\text { space index or element index during navigation }
\end{aligned}
$$

\footnotetext{
* Senior Researcher, Department of Engineering Science, University of Oxford

${ }^{\dagger}$ Associate Professor, Department of Engineering Science, University of Oxford
} 


$$
\begin{aligned}
& I \quad=\text { Imaginary unit } \sqrt{-1} \\
& j=\text { space index or element index during navigation } \\
& k=\text { passage index or space index during navigation } \\
& l=\text { harmonic index during navigation } \\
& n=\text { iteration index of pseudo time marching } \\
& N=\text { number of passages in an assembly } \\
& N_{h} \quad=\text { number of harmonics } \\
& p \quad=\text { pressure } \\
& \mathbf{q}=\text { primitive flow variable } \\
& R \quad=\text { gas constant } \\
& \mathbf{R}=\text { residual } \\
& S \quad=\quad \text { strain rate } \\
& \mathbf{S} \quad=\text { face normal } \\
& t=\text { time } \\
& T \quad=\text { temperature } \\
& T_{\max }=\text { maximum blade thickness } \\
& u \quad=\text { velocity } \\
& \mathbf{U}=\text { conservative variable } \\
& \mathbf{x}=\text { coordinate } \\
& \delta_{i j} \quad=\quad \text { Kronecker delta } \\
& \varepsilon=\text { coordinate in computational space } \\
& \rho \quad=\text { density } \\
& \kappa=\text { heat conduction coefficient } \\
& \tau_{i j}=\text { laminar shear stress tensor } \\
& \tau_{i j}^{t}=\text { Reynolds shear stress tensor } \\
& \phi \quad=\text { dummy variable } \\
& \psi \quad=\text { dummy variable } \\
& \omega=\text { angular frequency } \\
& \Omega \quad=\text { Volume }
\end{aligned}
$$

Subscripts and superscripts

' $=$ deterministic fluctuation to the passage-averaged mean flow

" = deterministic fluctuation to the density-averaged mean flow 


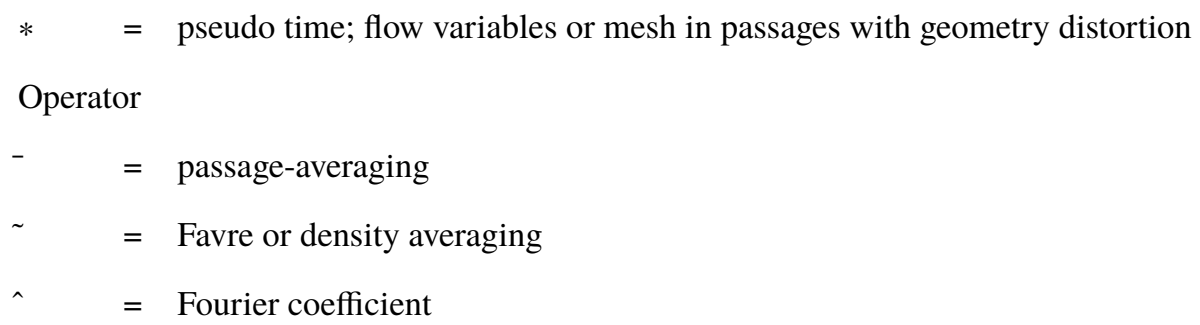

\section{Introduction}

Numerical simulations have been widely used in aerodynamic design, however, despite the advances in high performance computing, there are still scenarios where three dimensional (3D) steady Reynolds Averaged Navier-Stokes (RANS) simulations are too expensive to be used at the design stage, such as the shape optimization of an assembly of components in fluid machinery (e.g. gas turbine engines). It is common that each component or passage of an assembly could have mild differences in the geometry compared to neighbouring ones, in order to serve a certain design intent.

If all the components of an assembly have the same geometry, with the same boundary conditions, the flow in each passage is identical. The flow field in the assembly is periodic in the circumferential direction and the period is equal to the pitch of each component. In this case, only a single passage domain is required to model the assembly. If there are geometry distortions in the assembly, the flow is aperiodic in the circumferential direction. The whole assembly needs to be computed. Strictly speaking the flow in an assembly is always periodic with a period of $2 \pi$, but this is not of any practical interest. The term "aperiodic" in this work means the flow field in each passage is different from each other due to geometry distortions.

For example, the Outlet Guide Vane (OGV) in the bypass of a turbofan engine are designed in such a way that the blades in the OGV assembly exhibit a distortion in their geometries for the purpose of removing potential effects from downstream components (e.g. pylon) for the upstream fan blade. This treatment is critical to preserve the performance and integrity of the fan set. 3D RANS simulations of the whole OGV assembly in an iterative process is too computationally expensive to be used at the design stage for shape optimization. Therefore low fidelity models [1-4] have been developed by previous researchers based on potential flow theory. These low fidelity models are computationally efficient but with the penalty of ignoring viscous effects. In many cases these models can only be executed in 2D geometries, so 3D geometry effects (e.g. endwall blockage) might also be ignored. In order to enhance design confidence, there has been a growing need in the gas turbine industry to allow the shape optimization of an assembly to include 3D viscous effects in the design. Since CFD simulation is the most computationally intensive part of this iterative process, the bottleneck is how to devise an approach to significantly reduce the computational cost in the RANS simulations of the assembly with geometry distortions.

Several methods exist to reduce the computational cost in unsteady flows, such as the Non-Linear Harmonic (NLH) 
method by He and Ning [5] and the Harmonic Balance (HB) method by Hall et al [6]. Both methods exploit the periodicity of the flows, and they cast the unsteady Navier-Stokes (NS) equations as steady state problems in the frequency domain. The unsteady phenomenon is represented by a set of Fourier modes, which can be efficiently computed via pseudo-time marching, and an arsenal of techniques are available for convergence accelerations. Therefore periodic flows can be computed more efficiently in the frequency domain methods compared to the time-domain methods. The real reduction of the computational cost is realized when multiple components are simulated, such as multistage compressors. Unsteady RANS approach has to be executed on the whole wheel if a certain bladerow has odd number of passages. In contrast, the frequency domain method only requires one passage. This would normally lead to a reduction of computational cost by at least one order of magnitude. There has been extensive research on both methods for treating unsteady flows [6-12]. However, there is limited researches on geometry complexity using frequency domain methods. He [13] proposed an interesting approach to use spatial Fourier methods for steady and unsteady non-axi-symmetrical flows in the secondary air system of gas turbine engines, but in He's method, no geometry distortions is considered.

The contribution of this work is to devise a novel spatial Fourier method to efficiently simulate an assembly of components with geometry distortions. In this work, the relationship between grid displacement and flow perturbation is established via linear theory. For the assembly, the flow equations are linearzied around the passage-averaged flow. Due to the non-linearity of the NS equations, the passage-averaged flow differs from the single passage flow computed in the passage-averaged mean geometry. This difference is due to the presence of passage-averaged Deterministic Fluxes (DF) [14] in the governing equations of the passage-averaged flow, and this non-linear effect of DF is also demonstrated. Additionally, the paper demonstrates that the flow perturbation due to each harmonic geometry distortion can be used as basis solutions and they can be linearly combined to reconstruct flow perturbations with respect to new combinations of geometry distortions. This is perfectly suitable in an iterative design environment where new designs can be assessed in a timely manner.

This paper is organized as the following: Section $\Pi$ shows the principles of flow decomposition, the governing equations for the passage-averaged flow and the flow perturbations. Section [II] details the mathematical formulations of the proposed method. Section $[\mathrm{IV}$ provides the implementation details and this is followed by Section $\mathrm{V}$, which demonstrates the capability of the proposed method on a OGV assembly with flows in the transonic flow regime. Section $\mathrm{VI}$ concludes the paper. 


\section{Governing Equations}

The governing equation for the flow is the RANS equation and its differential form reads:

$$
\begin{gathered}
\frac{\partial \rho}{\partial t^{*}}+\frac{\partial\left(\rho u_{j}\right)}{\partial x_{j}}=0 \\
\frac{\partial\left(\rho u_{i}\right)}{\partial t^{*}}+\frac{\partial\left(\rho u_{i} u_{j}\right)}{\partial x_{j}}=-\frac{\partial p}{\partial x_{i}}+\frac{\partial}{\partial x_{j}}\left(\tau_{i j}+\tau_{i j}^{t}\right) \\
\frac{\partial(\rho E)}{\partial t^{*}}+\frac{\partial\left(\rho u_{j} H\right)}{\partial x_{j}}=-\frac{\partial}{\partial x_{j}}\left(\kappa \frac{\partial T}{\partial x_{j}}+u_{i}\left(\tau_{i j}+\tau_{i j}^{t}\right)\right)
\end{gathered}
$$

where $\kappa$ is the heat conduction coefficient. $\tau_{i j}^{t}$ is the Reynolds stress tensor. If Boussinesq approximation is used, $\tau_{i j}^{t}$ can be formulated using the local flow gradient at: $\tau_{i j}=2 \mu_{t}\left(S_{i j}-\frac{1}{3} \frac{\partial u_{k}}{\partial x_{k}} \delta_{i j}\right) . S_{i j}$ is the strain rate and is written as $\frac{1}{2}\left(\frac{\partial u_{i}}{\partial x_{j}}+\frac{\partial u_{j}}{\partial x_{i}}\right), \mu_{t}$ is turbulent viscosity and $\delta_{i j}$ is the Kronecker delta. The pseudo-time $t^{*}$ here has no physical meaning and it is simply a numerical treatment so that a steady state solution can be obtained via pseudo time marching.

For an assembly of components, assume there is a passage-mean or baseline geometry and the geometry of each passage is the linear combination of the baseline geometry and geometrical distortions (e.g. rigid body motion and re-cambering). The computational meshes for the components share the same mesh connectivity but the coordinates are slightly different due to the distortions of the geometries.

Let $\mathbf{x}_{k}$ denote the coordinates of the grid points in passage $k$. The coordinates of the mesh for the mean geometry $\overline{\mathbf{x}}$ can be defined as:

$$
\overline{\mathbf{x}}=\frac{1}{N} \sum_{k=1}^{k=N} \mathbf{x}_{k}
$$

Where $N$ is number of passages in the assembly. The grid displacement in passage $k$ relative to the passage-averaged mesh is defined as:

$$
\Delta \mathbf{x}_{k}=\mathbf{x}_{k}-\overline{\mathbf{x}}
$$

Similarly, the flow variables can be decomposed using Favre-averaging as the following [15, 16]: 


$$
\begin{aligned}
& u_{i}=\tilde{u}_{i}+u_{i}^{\prime \prime} \\
& \rho=\bar{\rho}+\rho^{\prime} \\
& p=\bar{p}+p^{\prime} \\
& h=\tilde{h}+h^{\prime \prime} \\
& e=\tilde{e}+e^{\prime \prime} \\
& T=\tilde{T}+T^{\prime \prime}
\end{aligned}
$$

Where the bar "-" represents passage averaging and the tilde " $\sim$ " Favre-averaging or density averaging. Their definitions for a dummy variable $\phi$ is defined as:

$$
\begin{aligned}
& \bar{\phi}=\frac{1}{N} \sum_{k=1}^{k=N} \phi_{k} \\
& \tilde{\phi}=\frac{\overline{\rho \phi}}{\bar{\rho}}
\end{aligned}
$$

If the flow decomposition in Equation 4 is substituted into Equation 1 followed by passage averaging, the governing equations for the passage-averaged flow can be obtained and it reads:

$$
\begin{aligned}
\frac{\partial \bar{\rho}}{\partial t^{*}}+\frac{\partial\left(\bar{\rho} \tilde{u}_{j}\right)}{\partial x_{j}} & =0 \\
\frac{\partial\left(\bar{\rho} \tilde{u}_{i}\right)}{\partial t^{*}}+\frac{\partial}{\partial x_{j}}\left(\bar{\rho} \tilde{u}_{i} \tilde{u}_{j}+\overline{\rho u_{i}^{\prime \prime} u_{j}^{\prime \prime}}\right) & =-\frac{\partial \bar{p}}{\partial x_{i}}+\frac{\partial}{\partial x_{j}}\left(\tilde{\tau}_{i j}+\tilde{\tau}_{i j}^{t}\right) \\
\frac{\partial}{\partial t^{*}}(\bar{\rho} \tilde{E})+\frac{\partial}{\partial x_{i}}\left(\bar{\rho} \tilde{u}_{i} \tilde{H}+\overline{\rho u_{i}^{\prime \prime} H^{\prime \prime}}\right) & =-\frac{\partial}{\partial x_{i}}\left(\kappa \frac{\partial \tilde{T}}{\partial x_{i}}\right)+\frac{\partial}{\partial x_{j}}\left[\tilde{u}_{i}\left(\tilde{\tau}_{i j}+\tilde{\tau}_{i j}^{t}\right)\right]+\frac{\partial}{\partial x_{j}}\left[\overline{u_{i}^{\prime \prime} \tau_{i j}^{\prime \prime}}+\overline{u_{i}^{\prime \prime} \tau_{i j}^{t \prime \prime}}\right]
\end{aligned}
$$

The extra terms which appear in the momentum and energy equations are deterministic fluxes. The existence of these terms are due to the flow variation in each passage and the non-linearity of NS equations when passage-averaging is performed [14].

The governing equations for the flow perturbations can be obtained by substituting the flow decomposition in Equation 4 into Equation 1 and then subtracting this equation by Equation 6 . If only the linear terms are collected, the governing equations of the linearized flow perturbations read: 


$$
\begin{aligned}
\frac{\partial \bar{\rho}}{\partial t^{*}}+\frac{\partial\left(\rho u_{j}\right)^{\prime \prime}}{\partial x_{j}} & =0 \\
\frac{\partial}{\partial t^{*}}\left(\rho u_{i}\right)^{\prime \prime}+\frac{\partial}{\partial x_{j}}\left(\left(\rho u_{i}\right)^{\prime \prime} \tilde{u}_{j}+\bar{\rho} \tilde{u}_{i} u_{j}^{\prime \prime}\right) & =-\frac{\partial p^{\prime}}{\partial x_{i}}+\frac{\partial}{\partial x_{j}}\left(\tau_{i j}^{\prime \prime}+\tau_{i j}^{t \prime \prime}\right) \\
\frac{\partial}{\partial t^{*}}(\rho E)^{\prime \prime}+\frac{\partial}{\partial x_{j}}\left(\left(\rho u_{j}\right)^{\prime \prime} \tilde{H}+\bar{\rho} \tilde{u}_{j} H^{\prime \prime}\right) & =-\frac{\partial}{\partial x_{j}}\left(\kappa \frac{\partial T^{\prime \prime}}{\partial x_{j}}\right)+\frac{\partial}{\partial x_{j}}\left[\tilde{u}_{i}\left(\tau_{i j}^{\prime \prime}+\tau_{i j}^{t \prime}\right)+u_{i}^{\prime \prime}\left(\tilde{\tau}_{i j}+\tilde{\tau}_{i j}^{t}\right)\right]
\end{aligned}
$$

The perturbations of the conservative variables (e.g. $\left.\left(\rho u_{i}\right)^{\prime \prime}\right)$ in Equation 7 are defined as:

$$
(\rho \phi)^{\prime \prime}=\bar{\rho} \phi^{\prime \prime}+\tilde{\phi} \rho^{\prime}
$$

Where $\phi$ is a dummy variable. Frozen turbulence is assumed in this paper to avoid tedious linearization of turbulence models, and the flow linearization is not applied on eddy viscosity $\mu_{t}$. The linearized turbulent shear stress is computed as: $\tau_{i j}^{t \prime \prime}=2 \mu_{t}\left(S^{\prime \prime}{ }_{i j}-\frac{1}{3} \frac{\partial u_{k}^{\prime \prime}}{\partial x_{k}} \delta_{i j}\right)$.

As is demonstrated by Wang and di Mare [12] it is advantageous to formulate the governing equations of the mean flow (either time-averaged or passage-averaged) using Favre-averaging, so that the formulations of the governing equations of the passage-averaged flow is identical to the one of the governing equation of the single passage flow apart from the extra contributions of deterministic fluxes.

\section{Methodology of Model Reduction}

\section{A. Grid Displacement and Flow Perturbation}

Assume there are two converged steady-state flow solutions using identical boundary conditions and mesh connectivity, but the coordinates of both meshes are slightly different due to a mild difference in the geometry. This is shown in Fig 1. The grid coordinate in the first mesh (baseline mesh) is denoted as $\mathbf{x}$ and the one in second mesh (perturbed mesh) is denoted as $\mathbf{x}^{*}$. Due to the difference in grid coordinate, the solutions on both meshes will be different. The baseline solution is denoted as $\mathbf{U}=\left[\rho, \rho u_{i}, \rho E\right]^{\mathrm{T}}$ and the perturbed one is denoted as $\mathbf{U}^{*}=\left[\rho^{*},(\rho u)_{i}^{*},(\rho E)^{*}\right]^{\mathrm{T}}$. The connections of both solutions to the difference in grid coordinates can be established in the following way.

The residuals of these two converged steady state solutions on meshes with coordinates $\mathbf{x}$ and $\mathbf{x}^{*}$ can be written as:

$$
\begin{aligned}
& \mathbf{R}(\mathbf{x}, \mathbf{U})=\mathbf{0} \quad \text { Baseline mesh } \\
& \mathbf{R}\left(\mathbf{x}^{*}, \mathbf{U}^{*}\right)=\mathbf{0} \quad \text { Perturbed mesh }
\end{aligned}
$$



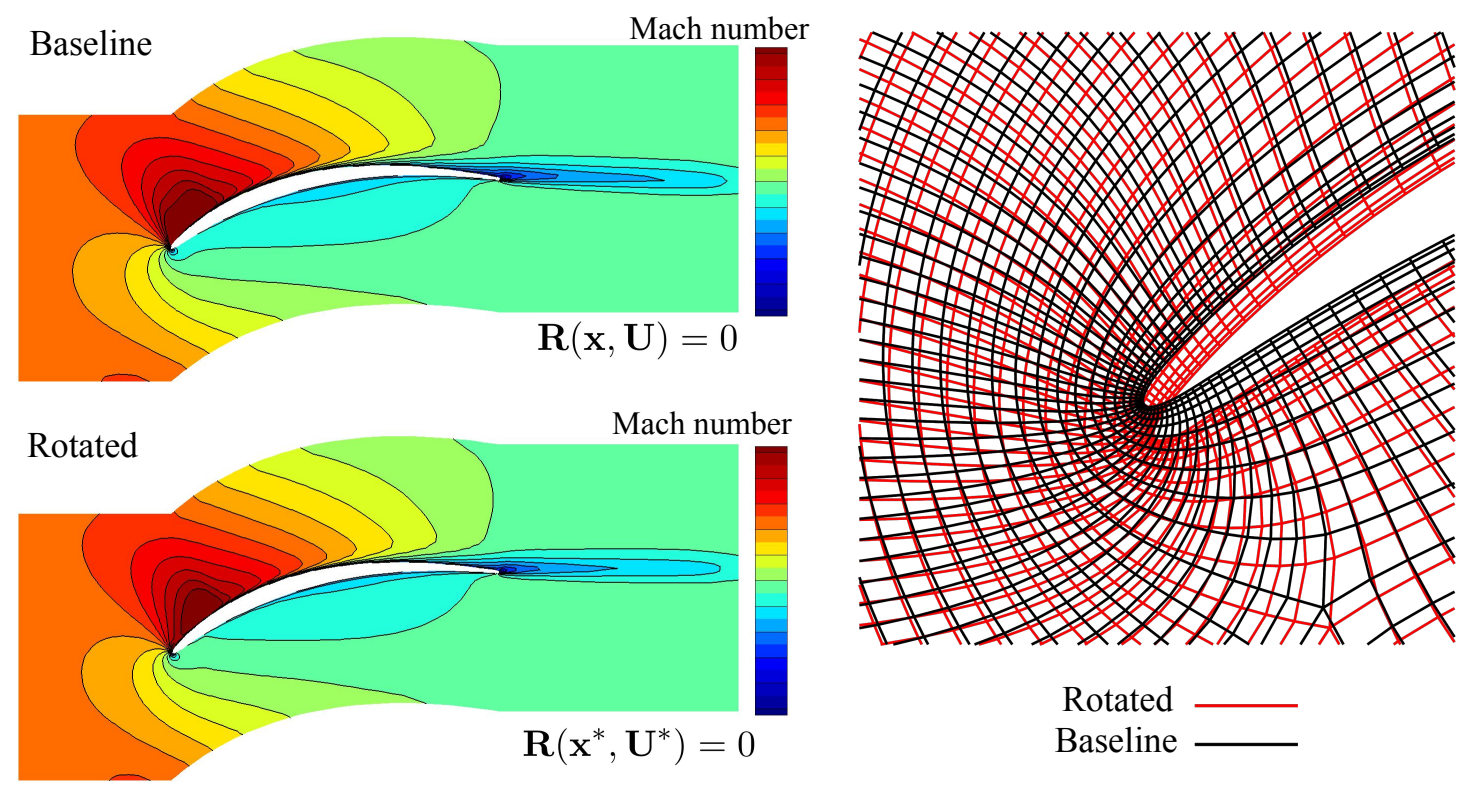

Rotated

Baseline

Fig. 1 Illustration of grid displacement and difference in flow fields.

The differences of the coordinate and flow solutions are defined as:

$$
\begin{aligned}
\Delta \mathbf{x} & =\mathbf{x}^{*}-\mathbf{x} \\
\Delta \mathbf{U} & =\mathbf{U}^{*}-\mathbf{U}
\end{aligned}
$$

$\Delta \mathbf{x}$ will be referred to as grid displacement and $\Delta \mathbf{U}$ flow perturbations, because the second solution $\mathbf{U}^{*}$ can be considered as being perturbed from the original solution $\mathbf{U}$. The relationship between $\Delta \mathbf{x}$ and $\Delta \mathbf{U}$ can be established as follows.

With the assumption of mild geometry distortion, $\mathbf{R}\left(\mathbf{x}^{*}, \mathbf{U}^{*}\right)$ can be expanded using Taylor series, and if only the linear terms are collected, such expansion reads:

$$
\mathbf{R}\left(\mathbf{x}^{*}, \mathbf{U}^{*}\right)=\mathbf{R}(\mathbf{x}, \mathbf{U})+\left.\frac{\partial \mathbf{R}}{\partial \mathbf{x}}\right|_{(\mathbf{x}, \mathbf{U})} \Delta \mathbf{x}+\left.\frac{\partial \mathbf{R}}{\partial \mathbf{U}}\right|_{(\mathbf{x}, \mathbf{U})} \Delta \mathbf{U}
$$

Since $\mathbf{R}\left(\mathbf{x}^{*}, \mathbf{U}^{*}\right)=\mathbf{0}$ and $\mathbf{R}(\mathbf{x}, \mathbf{U})=\mathbf{0}$, Equation 14 can be rewritten as:

$$
\left.\frac{\partial \mathbf{R}}{\partial \mathbf{U}}\right|_{(\mathbf{x}, \mathbf{U})} \Delta \mathbf{U}=-\left.\frac{\partial \mathbf{R}}{\partial \mathbf{x}}\right|_{(\mathbf{x}, \mathbf{U})} \Delta \mathbf{x}
$$

Equation 14 reveals the relationship between flow perturbation $\Delta \mathbf{U}$ and grid displacement $\Delta \mathbf{x}$. This relation will be extended for an assembly of components in the following section. 

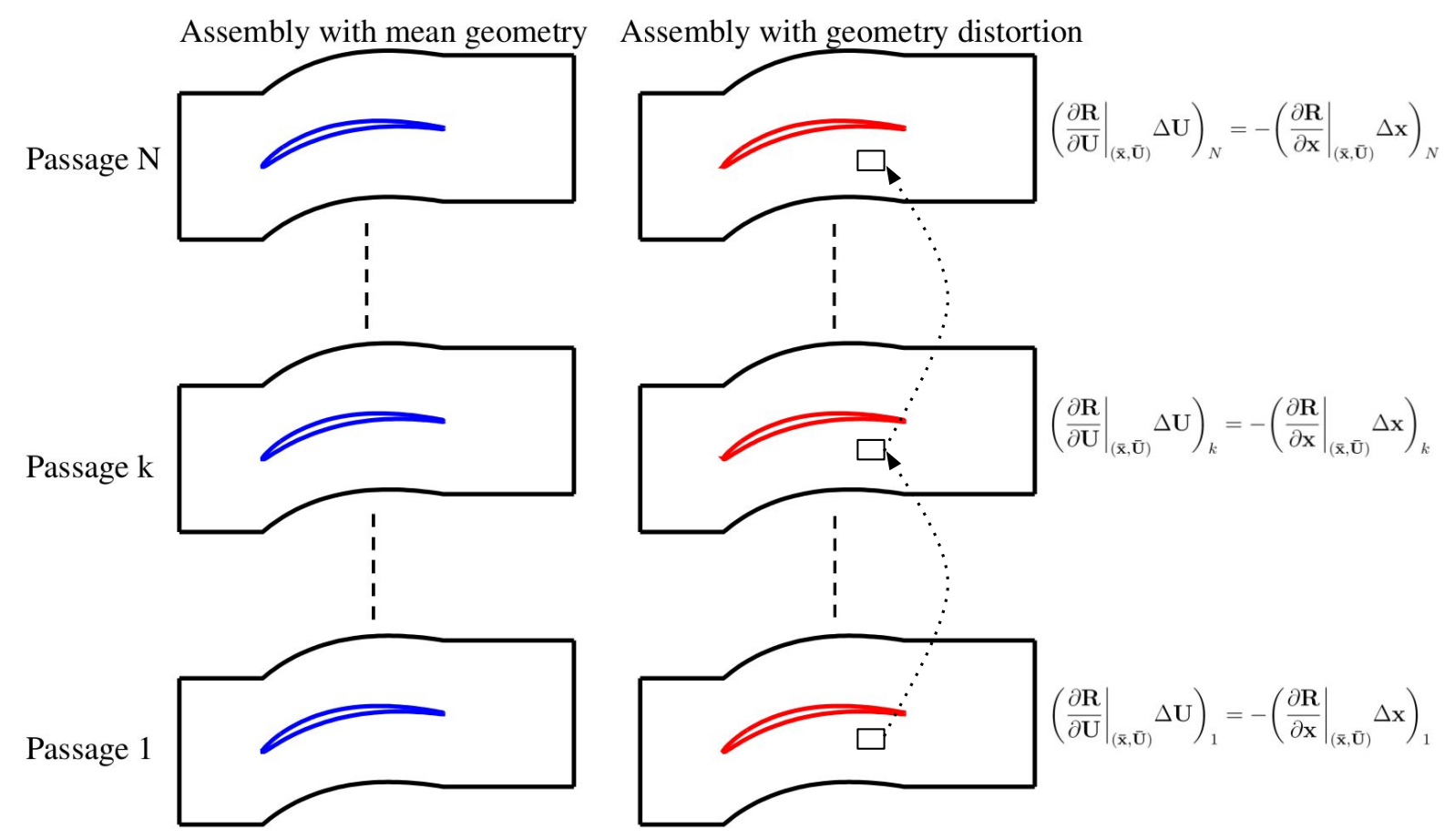

Fig. 2 Relating grid displacement with flow perturbations in an assembly.

\section{B. Model Reduction for Multiple Passages}

In an assembly, the grid displacement is defined as the difference between the grid coordinate in a certain component or passage and the one in the mean grid. This is defined in Equation 2 The flow perturbation is defined as the difference between the flow in a certain passage and the passage-averaged flow, which is described in Equation 4 and Equation 5 .

The relation between grid displacement and flow perturbations in Equation 14 can then be readily extended to an assembly of components and this is illustrated in Figure 2. The components on the left column represent a virtual assembly where each component has the passage-averaged grid and the passage mean flow. The components on the right shows a real assembly with geometry distortion and the flows in each passage is different. The passage-averaged conservative variable $\overline{\mathbf{U}}=\left[\bar{\rho}, \overline{\rho u_{i}}, \overline{\rho E}\right]$ is used for the formulation but it can be easily converted to the Favre-averaged primitive variables $\tilde{\mathbf{q}}=\left[\bar{\rho}, \tilde{u}_{i}, \bar{p}\right]$. For a perfect gas model, this conversion reads:

$$
\begin{aligned}
& \tilde{u}_{i}=\frac{\overline{\rho u_{i}}}{\bar{\rho}} \\
& \bar{p}=(\gamma-1) \overline{\rho e}=(\gamma-1) \bar{\rho}\left[\tilde{E}-\frac{1}{2}\left(\tilde{u}_{i} \tilde{u}_{i}+\overline{u_{i}^{\prime \prime} u_{i}^{\prime \prime}}\right)\right]
\end{aligned}
$$

where $\overline{\rho u_{i}^{\prime \prime} u_{i}^{\prime \prime}}$ is approximated as $\bar{\rho} \overline{u_{i}^{\prime \prime} u_{i}^{\prime \prime}}$ by ignoreing the triple terms $\overline{\rho^{\prime} u_{i}^{\prime \prime} u_{i}^{\prime \prime}}$. 
Assume the grid displacement $\Delta \mathbf{x}_{k}$ is known for each passage, for an assembly of components Equation 14 can be written as:

$$
\left(\left.\frac{\partial \mathbf{R}}{\partial \mathbf{U}}\right|_{(\overline{\mathbf{x}}, \overline{\mathbf{U}})} \Delta \mathbf{U}\right)_{k}=-\left(\left.\frac{\partial \mathbf{R}}{\partial \mathbf{x}}\right|_{(\overline{\mathbf{x}}, \overline{\mathbf{U}})} \Delta \mathbf{x}\right)_{k}
$$

Where $k$ is the passage index. Since the Jacobian matrix $\left.\frac{\partial \mathbf{R}}{\partial \mathbf{U}}\right|_{(\overline{\mathbf{x}}, \overline{\mathbf{U}})}$ and $\left.\frac{\partial \mathbf{R}}{\partial \mathbf{x}}\right|_{(\overline{\mathbf{x}}, \overline{\mathbf{U}})}$ only contain the flow variable and coordinate of the mean geometry, they are independent of the passage index, therefore Equation 16 can be rewritten as:

$$
\left.\frac{\partial \mathbf{R}}{\partial \mathbf{U}}\right|_{(\overline{\mathbf{x}}, \overline{\mathbf{U}})} \Delta \mathbf{U}_{k}=-\left.\frac{\partial \mathbf{R}}{\partial \mathbf{x}}\right|_{(\overline{\mathbf{x}}, \overline{\mathbf{U}})} \Delta \mathbf{x}_{k}=\mathbf{G}_{k}
$$

The right hand side $-\left.\frac{\partial \mathbf{R}}{\partial \mathbf{x}}\right|_{(\overline{\mathbf{X}}, \overline{\mathbf{U}})} \Delta \mathbf{x}_{k}$ is essentially a source terms and it leads to flow perturbations due to the grid displacement, which is a result of geometry distortion. If $\mathbf{x}_{k}$ is zero, $\Delta \mathbf{U}_{k}$ will be reduced to zero. This means the flow in each passage is the same and is also identical to the passage mean flow. For simplicity, $-\left.\frac{\partial \mathbf{R}}{\partial \mathbf{x}}\right|_{(\overline{\mathbf{x}}, \overline{\mathbf{U}})} \Delta \mathbf{x}_{k}$ is represented by the symbol $\mathbf{G}_{k}$ in the following text.

The term $\mathbf{G}_{k}$ is non-trivial to evaluate, since it involves the linearization of the fluxes with respect to the grid displacement. For example, it is typical that the convective flux is computed using an upwind scheme with the Roe's approximate Riemann solver, which can be written as:

$$
\mathbf{F}_{m}=\frac{1}{2}\left[\mathbf{F}_{L}+\mathbf{F}_{R}-\left|\mathbf{A}_{\text {Roe }}\right|\left(\mathbf{U}_{\mathrm{R}}-\mathbf{U}_{\mathrm{L}}\right)\right] \| \mathbf{S}||
$$

$\mathbf{F}_{m}$ represents the numerical fluxes across the cell interface. $\mathbf{F}_{L}$ and $\mathbf{F}_{R}$ represent the physical fluxes on the left and right of the cell interface. $\left|\mathbf{A}_{\text {Roe }}\right|$ is the Roe matrix [17], which is evaluated by the Roe's averages. $\|\mathbf{S}\|$ is the area of the cell interface. The grid displacement will stretch the face area and rotate the face normal. It is noted that the normal of the cell interface is used both in the physical fluxes and also in the Roe matrix, and linearization of the Roe matrix with respect to the grid displacement is required to compute $\mathbf{G}_{k}$. From a practical point of view, this will involve considerable code development, therefore we need an alternative way to compute $\mathbf{G}_{k}$.

Since the grid displacement is known, with considerably less effort, $\mathbf{G}_{k}$ can be computed directly using finite difference by ignoring the high order terms, i.e.:

$$
\mathbf{G}_{k}=\mathbf{R}_{\mathbf{k}}\left(\mathbf{x}_{\mathbf{k}}, \overline{\mathbf{U}}\right)-\mathbf{R}_{\mathbf{k}}(\overline{\mathbf{x}}, \overline{\mathbf{U}})=\left.\frac{\partial \mathbf{R}}{\partial \mathbf{x}}\right|_{(\overline{\mathbf{x}}, \overline{\mathbf{U}})} \Delta \mathbf{x}_{k}
$$

Basically $\mathbf{G}_{k}$ can be easily computed by calling the existing routines to assemble the right hand side of a RANS solver twice. The first time is for the mean geometry and the second time is for the right hand side with the perturbed mesh. This will save the effort of implementing the linearizing flux with respect to the grid displacement $\Delta \mathbf{x}_{k}$ and have 
minimum implemention effort on the existing CFD solver.

Assume the geometry distortion in the assembly can be decomposed into a set of discrete Fourier series [4]. A harmonic variation of the grid coordinate on the blade surface will lead to a harmonic variation of the grid coordinate in the interior of each passage. The difference of the grid coordinate in adjacent passages is linked by the InterBlade Phase Angle (IBPA) of the harmonic geometry distortion. Equation 17 shows the relation between grid displacement and flow perturbations in each passage, therefore the harmonic grid displacement will lead to a harmonic flow perturbation with the same IBPA.

A Fourier transform (FT) can be applied on both sides of Equation 17 and this will lead to a set of equations corresponding to each harmonic of the geometry distortion. Since each passage has identical mesh connectivity, the $i^{t h}$ cell of each passage has unique corresponding cells in other passages, which is illustrated in Fig. 2. If FT of the $i^{t h}$ cell of $\mathbf{G}_{k}$ is to be computed, the Fourier transform $\mathcal{F}\left(\mathbf{G}_{k}^{i}\right)$ is applied on the data set $\mathbf{G}_{k}^{i}$, which consists of:

$$
\left\{\mathbf{G}_{k}^{i}\right\}=\left\{\mathbf{G}_{1}^{i}, \mathbf{G}_{2}^{i}, \ldots, \mathbf{G}_{N}^{i}\right\}
$$

Where $k$ is the passage index. The FT for the flow variations $\mathcal{F}\left(\Delta \mathbf{U}_{k}^{i}\right)$ are conducted in an identical fashion. It is noted that FT is performed on cell or node index instead of using their actual coordinates. Therefore an uniform data sampling for FT always hold. After the Fourier transform, without losing generality, if only one harmonic of the geometry distortion is considered here. the equation for harmonic index $l$ can be written as:

$$
\left.\frac{\partial \mathbf{R}}{\partial \mathbf{U}}\right|_{(\overline{\mathbf{x}}, \overline{\mathbf{U}})} \hat{\mathbf{U}}_{l}=\hat{\mathbf{G}}_{l}
$$

where: $\hat{\mathbf{U}}_{l}$ is the $l^{t h}$ harmonic of $\mathcal{F}\left(\mathbf{U}_{k}\right) . \hat{\mathbf{G}}_{l}$ is the $l^{t h}$ harmonic of $\mathcal{F}\left(\mathbf{G}_{k}\right)$. By adding a pseudo time $t^{*}$ on the left hand side, Equation 21 can be solved with classical iterative techniques for steady state problems, such as Runge-Kutta.

$$
\frac{\partial \hat{\mathbf{U}}_{l}}{\partial t^{*}}+\left.\frac{\partial \mathbf{R}}{\partial \mathbf{U}}\right|_{(\overline{\mathbf{x}}, \overline{\mathbf{U}})} \hat{\mathbf{U}}_{l}=\hat{\mathbf{G}}_{l}
$$

It is interesting to note that there is no spectral source terms (e.g. I $\left.\omega_{l} \hat{\mathbf{U}}\right)$ in Equation 22 From the point of the view of the linearized unsteady flows, Equation 22 can be considered as the one which is solving a disturbance with an extra source term $\hat{\mathbf{G}}_{l}$ but with zero frequency.

\section{Solution Method}

The proposed method has been implemented in the in-house CFD solver AU3X [18, 19]. AU3X solves the unsteady Reynolds-Averaged Navier-Stokes (RANS) equations implicitly on unstructured meshes using a cell-centered finite volume scheme. The code is formally second-order accurate in space and time. The flow gradients are evaluated 
using weighted least square method [20] and convective fluxes are computed by Roe's approximated Riemann solver. Monotonic Upwind Scheme for Conservation Law (MUSCL) is used for the upwind fluxes with the van Albada limiter. The code has also recently been optimized on modern many-core architectures [21].

For the linearized solver, the gradient of the Fourier coefficients are computed with the weighted least square method. Second order spatial accuracy is achieved by interpolating the flow perturbations, which are in the form of complex numbers, from the cell centers to the cell interface using the MUSCL scheme with the Van Albada limiter.

The resulting coupled system of the passage-averaged mean flow (Equation 6 ) and the linearized NS (Equation 7 ) for the $l^{\text {th }}$ harmonic is shown in Equation 23 .

$$
\begin{aligned}
& \left(\frac{\Omega_{i}}{\Delta t^{*}} \delta_{i j}+\left.\sum_{j=1}^{j=N_{e}} \frac{\partial \mathbf{R}_{i j}(\mathbf{U})}{\partial \mathbf{U}_{\mathbf{i}}}\right|_{\mathbf{U}_{\mathbf{i}}^{\mathbf{n}}}\right) \Delta \mathbf{U}_{\mathbf{i}}^{\mathbf{n}}=\sum_{j=1}^{j=N_{e}}-\mathbf{R}_{i j}\left(\mathbf{U}^{\mathbf{n}}\right) \\
& \left(\frac{\Omega_{i}}{\Delta t^{*}} \delta_{i j}+\left.\sum_{j=1}^{j=N_{e}} \frac{\partial \mathbf{R}_{i j}(\mathbf{U})}{\partial \mathbf{U}_{\mathbf{i}}}\right|_{\mathbf{U}_{i}^{n}}\right) \Delta \hat{\mathbf{U}}_{\mathbf{i}}^{\mathbf{n}}=\sum_{j=1}^{j=N_{e}}\left(-\left.\frac{\partial \mathbf{R}_{i j}(\mathbf{U})}{\partial \mathbf{U}_{\mathbf{i}}}\right|_{\mathbf{U}_{i}^{n}} \hat{\mathbf{U}}_{i}^{n}\right)+\hat{\mathbf{G}}_{l}
\end{aligned}
$$

Where $i, j$ is the cell index, $\Omega_{i}$ is the volume of cell $i, \delta_{i j}$ is the Kronecker delta, $n$ is the time step, $\Delta \mathbf{U}_{i}^{n}=\mathbf{U}_{i}^{n+1}-\mathbf{U}_{i}^{n}$ and $\Delta \hat{\mathbf{U}}_{i}^{n}=\hat{\mathbf{U}}_{i}^{n+1}-\hat{\mathbf{U}}_{i}^{n}, \mathbf{R}_{i j}$ is the flux flowing through the interface between cell $i$ and $j$.

Equation 23 shows a similar form to a typical NLH [5] or FNLH method [12]. The main difference is in the system of the linearized flow. The terms with respect to the spectral source term $\omega_{l} \Omega_{i} \hat{\mathbf{U}}_{i}^{n}$ is not present, due to the fact that the frequency in the linearized solver is zero. Besides, there is the extra term $\hat{\mathbf{G}}_{l}$ in the right hand side in the linearized solver, which represents the contribution of grid displacement.

$\left.\sum_{j=1}^{j=N_{e}} \frac{\partial R_{i j}(\mathbf{U})}{\partial \mathbf{U}_{i}}\right|_{\mathbf{U}_{i j}^{n}}$ is the flux Jacobian of cell $i$ accumulated on the interfaces with the neighboring cells. $N_{e}$ is the number of neighboring cells of cell $i$. Similar to our previous work [12], a matrix free approach is used and the flux Jacobian is approximated by its spectral radius. The left hand side matrix is then approximated by a diagonal matrix and effectively stored as an one-dimensional array. This requires less memory storage and also is easier to implement than storing the Jacobian matrix, but the trade-off is slower convergence rate [22]. The mean flow is solved implicitly with Newton-Jacobi and the linearized flow is solved with explicit 5-stage Runge-Kutta using the following coefficients [23] $\alpha_{i}=[0.0695,0.1602,0.2898,0.5060,1]$. This treatment is found to be robust for OGV blades in transonic flow regime under typical geometry modifications, such as restaggering ahd recambering.

It is noted that the evaluation of deterministic fluxes can be optionally ignored. The approach to be referred to as SFNLH (Spatial Faver-averaged Non-Linear Harmonic) model in this paper solves the equation for the passage average flow which includes the effect of flow variations from passage to passage. This variation is accounted for by the deterministic fluxes, which are evaluated from the solution of the linear perturbation equations. Thus both equations shown in Equation 23 are coupled with each other. If one assumes the base flow is the single passage solution of the 
unperturbed geometry, there is no coupling. The resulting model is referred to as the SLH (Spatial Linear Harmonic) model in this paper.

\section{A. Boundary Conditions}

In the current work the flow perturbation is related to grid displacement. The flow perturbations on the freestream boundaries (e.g. inlet and exit) are set to zero. The periodic and wall boundaries are handled as the following ways:

Periodic Boundary For the linearized solver of the flow perturbation, a phase shift is applied on the lower periodic boundary and the resulting values are applied to the upper periodic boundary. If the IBPA $\sigma$ is known, this can be formulated as:

$$
\hat{\mathbf{U}}_{\text {upper }}=\hat{\mathbf{U}}_{\text {lower }} e^{I \sigma}
$$

Since there is no spectral source terms in Equation 22, the coupling of the real part and the imaginary part only happens on the periodic boundaries via the phase shift term $e^{I \sigma}$.

Viscous Wall The mean flow and the linearized solver for the flow perturbation both use non-slip wall boundary conditions. Automatic wall treatment is used in the mean flow [24]. At each iteration, $y^{+}$is computed for the first cell on the wall and the wall shear stress is computed, which depends on whether the $y^{+}$is larger than 11 (wall function) or smaller than 11 (viscous sublayer). The linearized flow uses the $y^{+}$from the mean flow to compute the linearized wall shear stress on the wall. If $y^{+}$is larger than 11, the linearized wall function is used, otherwise the linearized viscous sublayer is used instead.

\section{B. Evaluation of Deterministic Fluxes}

Deterministic fluxes in Equation 6 can be computed as the following way. If the triple terms (e.g. $\overline{\rho^{\prime} u_{i}^{\prime \prime} u_{j}^{\prime \prime}}$ and $\left.\overline{\rho^{\prime \prime} u_{i}^{\prime \prime} H^{\prime \prime}}\right)$ are ignored, The deterministic fluxes can be written as: $\overline{\rho u_{i}^{\prime \prime} u_{j}^{\prime \prime}} \approx \bar{\rho} \overline{u_{i}^{\prime \prime} u_{j}^{\prime \prime}}$ and $\overline{\rho u_{i}^{\prime \prime} H^{\prime \prime}} \approx \bar{\rho} \overline{u_{i}^{\prime \prime} H^{\prime \prime}}$. In such a way, deterministic fluxes in Equation 6 are formulated as the passage average of the product of two perturbations. If the Fourier modes of both perturbations are known, the passage average of their products can be computed as:

$$
\overline{\phi^{\prime \prime} \psi^{\prime \prime}}=2 \sum_{l=1}^{l=N_{h}}\left[\operatorname{Re}\left(\hat{\phi}_{l}\right) \operatorname{Re}\left(\hat{\psi}_{l}\right)+\operatorname{Im}\left(\hat{\phi}_{l}\right) \operatorname{Im}\left(\hat{\psi}_{l}\right)\right]
$$

$l$ is the harmonic index, $N_{h}$ is the number of harmonics. $\phi$ and $\psi$ are dummy variables.

For a finite volume solver, deterministic fluxes on the cell interface are computed via inverse-distance weighted averaging and they will be accumulated to the right hand side and represent the contributions of deterministic fluxes on extra momentum and energy transfer. 


\section{Implementation Details and Computational Cost}

The major steps to implement the proposed method is described in Algorithm 1. The computational cost of the proposed method is similar to the NLH or FNLH methods. Computing one harmonic requires roughly the computational cost of two steady single-passage computations, one for the real part and one for the imaginary part of the flow perturbations. The extra cost compared to NLH or FNLH is the evaluation of the right hand side $\hat{\mathbf{G}}_{l}$ for the linearized flow, which takes into account the effect of grid displacement.

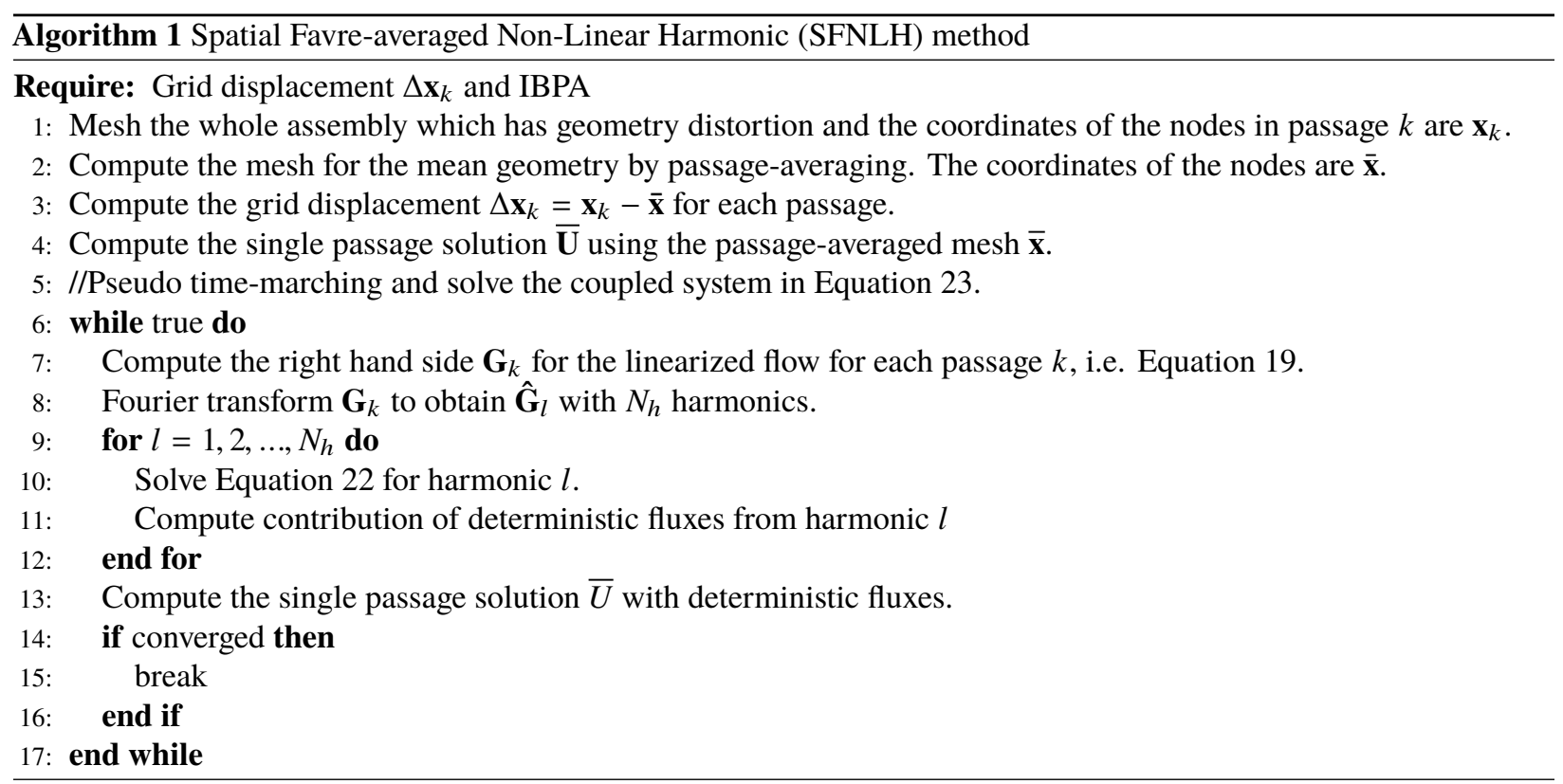

Suppose there are $N$ passages in the assembly, the computational cost to evaluate $\hat{\mathbf{G}}_{l}$ is $N$ times the computational cost to compute the right hand side for the steady state flow of a single passage plus one extra Fast Fourier Transform (FFT). Such computational cost is found to be comparable to or even more expensive than the ones for the mean flow computation and the linearized flow at each pseudo-time step. However, it it noted that the difference of the passage-averaged flow and the single passage flow is generally small. There will only be a minor difference between the Jacobian matrices $\frac{\partial \mathbf{R}}{\partial \mathbf{x}}$ using the single passage solution or the passage-averaged solution. Therefore computing $\frac{\partial \mathbf{R}}{\partial \mathbf{x}}$ can be safely skipped every 10 or 20 pseudo-time steps. This will offset its extra computational cost and is found to have a negligible effect on the robustness of the method.

An alternative solution is to perform a sampling of the solution in the assembly. The previous approach can be considered a special sampling case where all the passages are used for the sampling. In this approach, a non-uniform sampling might be required if number of harmonics and number of passages do not fit. This would introduce extra coding effort. Therefore the first approach is easier to implement and it is used in this work.

In certain situations (e.g. small grid displacement) the effect of deterministic fluxes are considered to be of secondary importance, SFNLH will reduce to SLH if no DF is included in the mean flow, and $\hat{\mathbf{G}}_{l}$ can be computed only once at the 

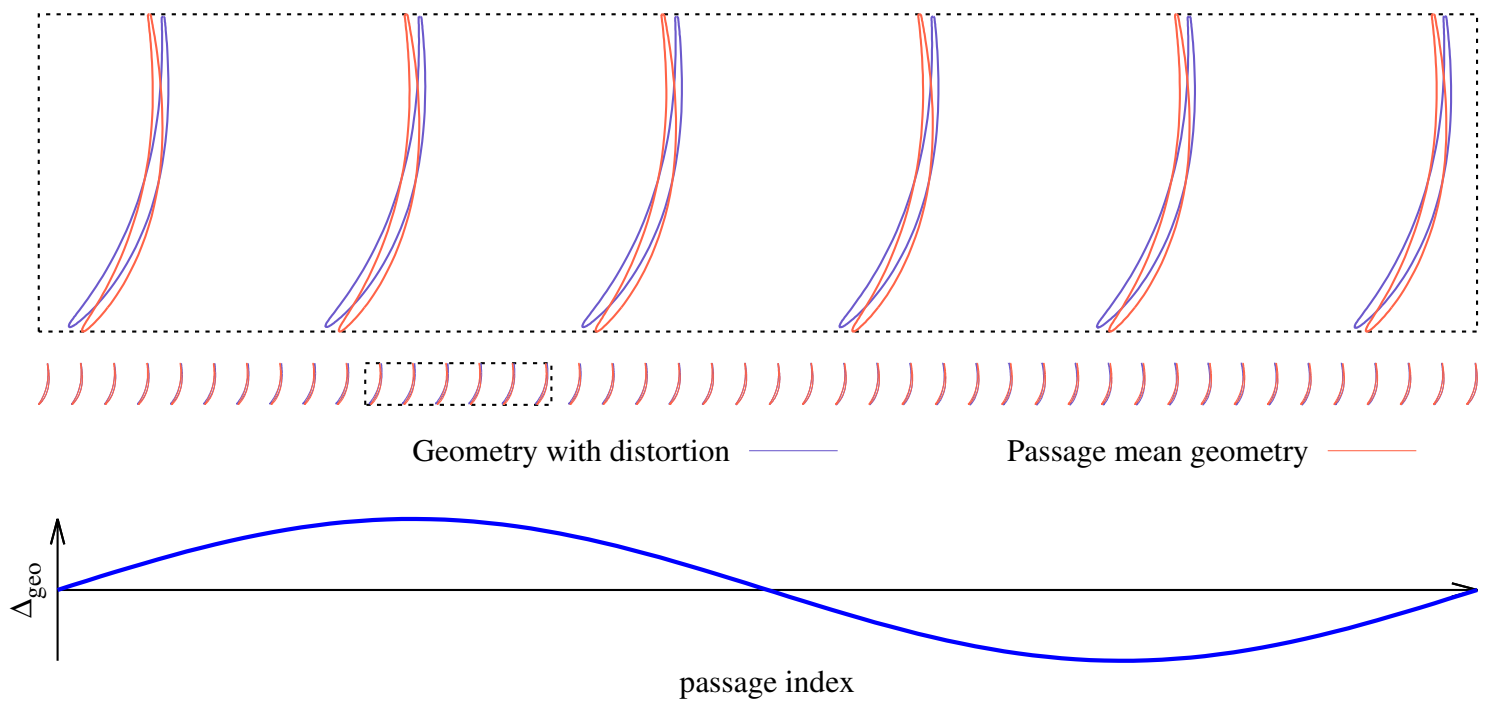

Fig. 3 Harmonic modification of blade geometry.

beginning of the computation, since $\overline{\mathbf{U}}$ and $\Delta \mathbf{x}$ come from the steady single passage solution and they are fixed during the computation.

Finally, the cost reduction of the proposed method is diminishing as $N_{h}$ increases or $N$ decreases. As an estimation, if the computational cost to compute a single steady solution is taken as unity, the computational cost to compute $N_{h}$ harmonic is roughly $2 N_{h}+\mathrm{FG}+1$. FG is the computational cost to compute $\hat{G}_{l}$, whose computational cost is a fraction of a single steady solution. If there are $N$ passages in the assembly, the saving in terms of computational cost compared to the whole assembly solution is $r=N /\left(2 N_{h}+\mathrm{FG}+1\right)$. Assume $N=44$ and $\mathrm{FG}=0.2$, if $N_{h}=1, r=13.75, r$ reduces to 3.92 when $N_{h}$ increases to 5 . Furthermore, if $N$ is reduced to 22 , the cost saving will be 6.87 and 1.96 respectively.

\section{Results}

The capability of the method is demonstrated on the middle span slice of the OGV assembly in a modern gas turbine engine. RANS/URANS simulations of the full 3D geometries have been validated against experimental data by Carnevale et al [25]. The focus here is to demonstrate that the proposed method can obtain a good reconstruction of the flow perturbations caused by geometry distortions compared to direct whole assembly simulations. Rotation and re-cambering are typical geometry distortions that are introduced in the OGV assembly. Without losing generality, only one harmonic of rotation and re-cambering is considered. The schematic is shown in Fig. 3 . Furthermore a parametric study of various levels of rotation and re-cambering is also performed to demonstrate the non-linear effect of deterministic fluxes. Finally the strategy to handle combined rotation and re-cambering is demonstrated and validated. 


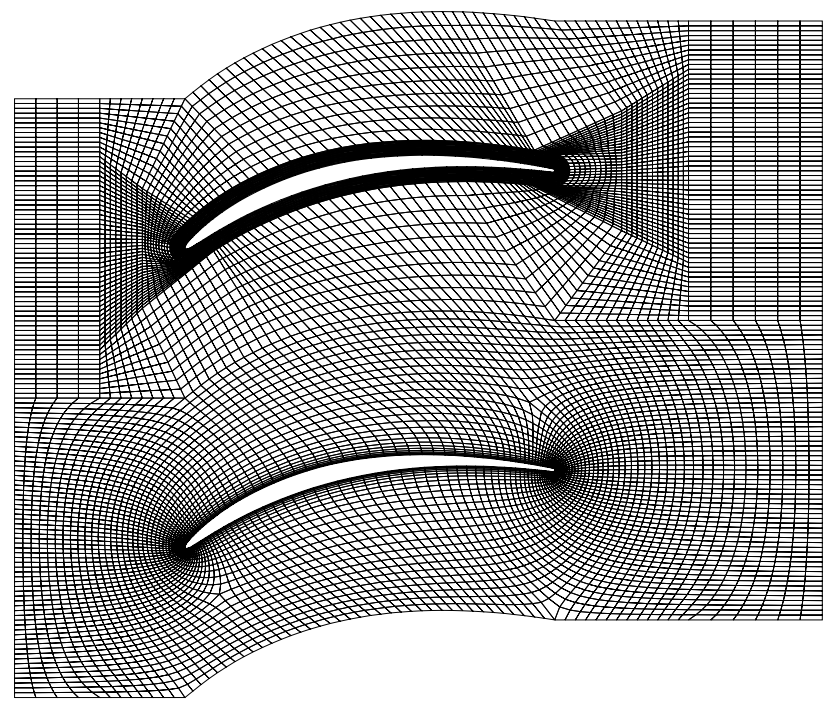

Fig. 4 Algebraic and elliptically smoothed meshes.

\section{A. Mesh Generation}

Multi-block structured mesh is used in this study. Elliptic smoothing is used to remove grid folding and compute boundary layer meshes on the blade. More details on this meshing technique can be found in Wang [26, 27]. Typical mesh used in this study is shown in Fig. 4, which shows the initial algebraic mesh (Top) and the final mesh (Bottom) after elliptic smoothing. If the whole assembly with geometry distortion is the case, the mesh in each passage is generated with identical mesh connectivity and elliptically smoothed to ensure the coordinates of the corresponding nodes in each passage change smoothly in the assembly. The periodic boundaries of each passage are fixed in the smoothing iteration and the periodic boundaries are fully conformal for adjacent passages of the whole assembly.

\section{B. Single Passage Solution and Grid Sensitivity Study}

The assembly has 44 airfoils and the baseline (or passage-mean) blade geometry has a pitch-chord ratio of 0.8 with a stagger angle of $12^{\circ}$. The Mach number distribution of a single passage solution on the mean geometry is shown in Fig. 5. The flow feature is the transonic flow patch on the suction side, where the flow is strongly non-linear.

Mesh sensitivity study is performed on the single passage computation with three sets of meshes. The coarse, medium and fine meshes have 6122, 11977 and 19190 cells respectively. The average $y^{+}$of the coarse mesh is around 30, and the ones of the medium and fine meshes are around 1. The distribution of Mach number computed by the medium mesh is shown in Fig. 5, where one can clearly see a transonic flow region on the suction side.

The computed pressure distribution using the coarse, medium and fine meshes are shown in Fig. 6 The values are normalized by the maximum pressure on the blade. It can be seen that the difference between the medium mesh and the fine one is marginal. The meshing parameters for the medium mesh are used in the following study and they will also be used for generating meshes for other passages with mild geometrical variations. 


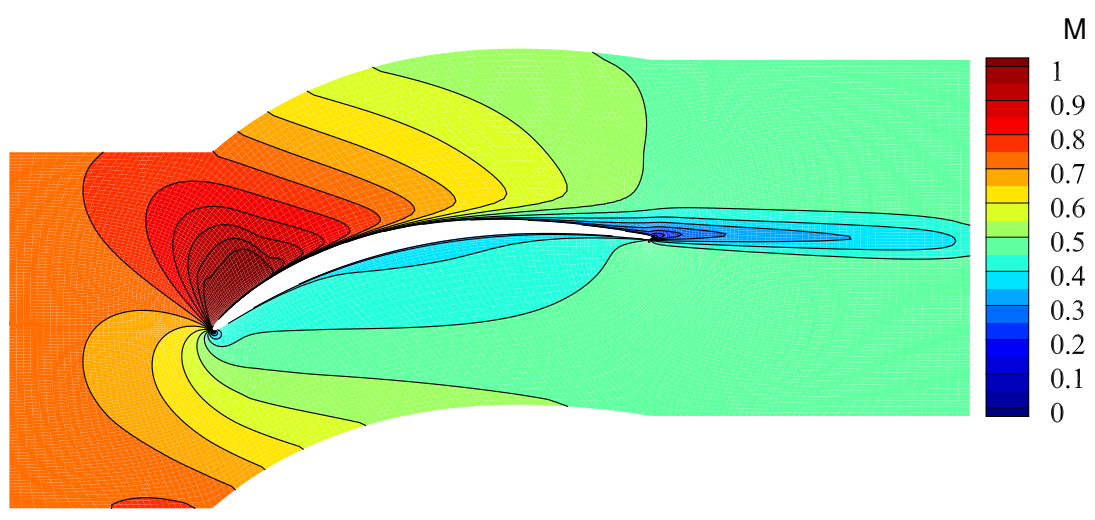

Fig. 5 Mach number distribution in the single passage simulation.

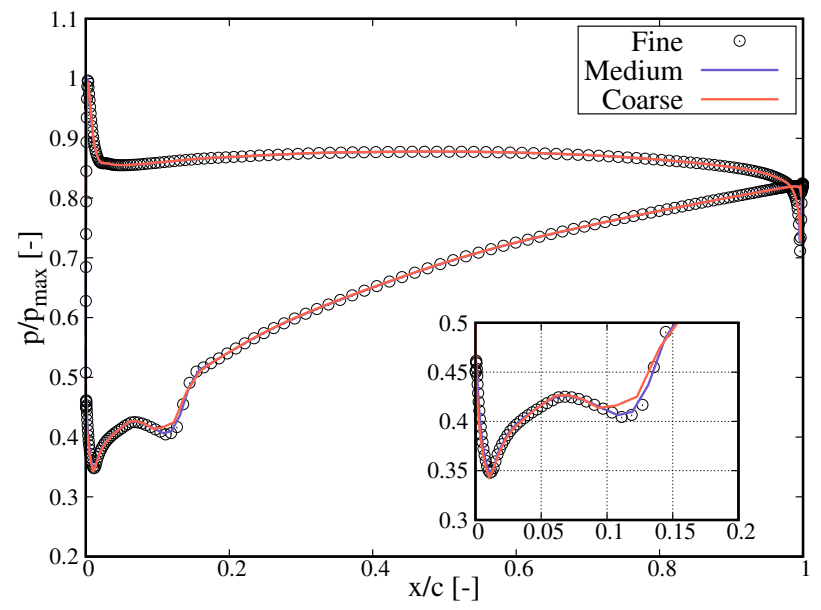

Fig. 6 Grid dependence study. 


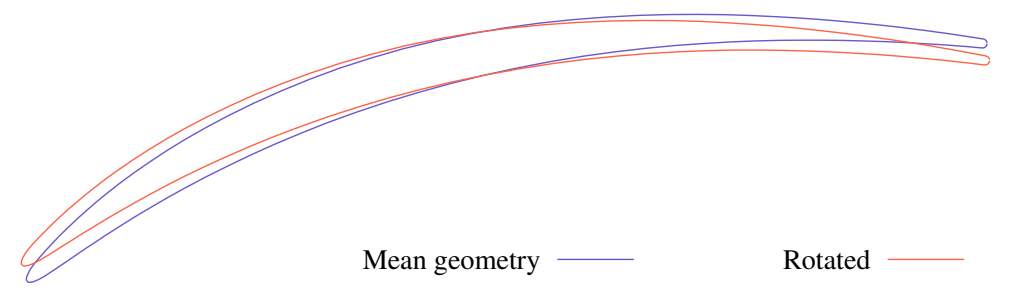

Fig. 7 Rotation of a blade.

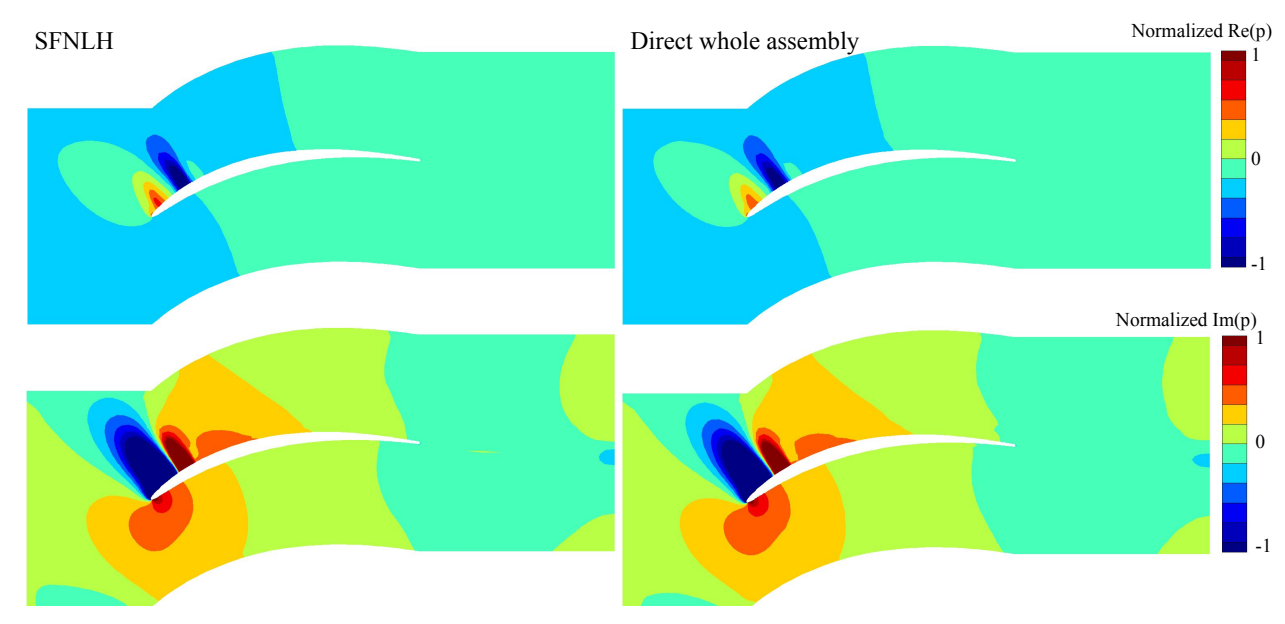

Fig. 8 Pressure variations in the passage with blade rotation.

\section{Rotation}

The first test case is a single harmonic rotation of the assembly with a wave length of $2 \pi$. The schematic is shown in Fig. 3 The IBPA of the geometry distortion between adjencent passage is $\frac{\pi}{22}$. Figure 7 shows the schematic of such harmonic rotation. The cascade is rotated harmonically by $0.5^{\circ}, 1^{\circ}$ and $2^{\circ}$ and the variations of pressure and velocity in the passages are examined in detail and compared with direct whole assembly solutions.

\section{Pressure Variation}

Since it is cumbersome to show the solutions of each passage, Fourier transform of the direct whole assembly solutions is performed and the results are rendered on the passage-averaged geometry. Figure 8 shows the distributions of real and imaginary part of pressure variations in the passage and its comparisons with the direct whole assembly solutions when the magnitude of the harmonic rotation is $1^{\circ}$. The values are normalized by the largest magnitude of the pressure variation in the domain. Good agreement with the direct solutions is observed for both the real and imaginary parts. The most noticeable feature of pressure variation is the pattern on the suction side close to the blade leading edge. This is because blade rotation will lead to the variation of the incidence, which leads to the shift of the transonic flow patch on the cascade harmonically in the assembly.

The distribution of the pressure perturbations on the blade is shown in Fig. 9 All the values are normalized by the by $p_{\text {ref }} \times \alpha$, where $p_{\text {ref }}$ is the largest $\operatorname{Im}(p)$ on the blade with a rotation angle of $2^{\circ}$ in the direct whole assembly solution 



Fig. 9 Pressure variations on the blade with blade rotation.

and $\alpha$ is the corresponding rotation angle. Such normalization will remove all the linear effect and reveal the non-linear effects of flow perturbations related to geometry modification. Good agreement with the direct solutions are observed despite the presence of the transonic flow region on the suction side. The results in Fig.9 9 also show that the variation of the blade loading is largest towards the leading edge and there is negligible loading variations towards the trailing edge.

Besides, Figure 9 clearly shows the non-linear effects on the blade surface for the pressure variations around the transonic region when the rotation angle is increased from $0.5^{\circ}$ to $2^{\circ}$. Away from the transonic region, the curves are overlapping with each other and this means a linear response of the pressure variation to blade rotation.

Due to geometry variation, the flow fields in each passage will be different from each other. The governing equations of the passage-averaged flow will contain deterministic fluxes due to the non-linearity of the NS equations. To demonstrate this non-linear effect, the pressure perturbations on the blades are compared between SLH and SFNLH solutions. This is shown in Fig. 10 and the values are normalized in the same way as Fig. 9. Such normalization removes all the linear effects and clearly shows the non-linear effect. SLH is found to be sufficiently accurate on the pressure side, which shows the pressure changes linearly with blade rotation angle. The non-linear effect of deterministic fluxes is clearly seen on $2 \%$ to $20 \%$ chord of the suction side, where the flow is transonic. Therefore SLH should be used with care if the flow is transonic. On the rest locations on the suction side, the linear response of pressure to blade rotation is observed. 

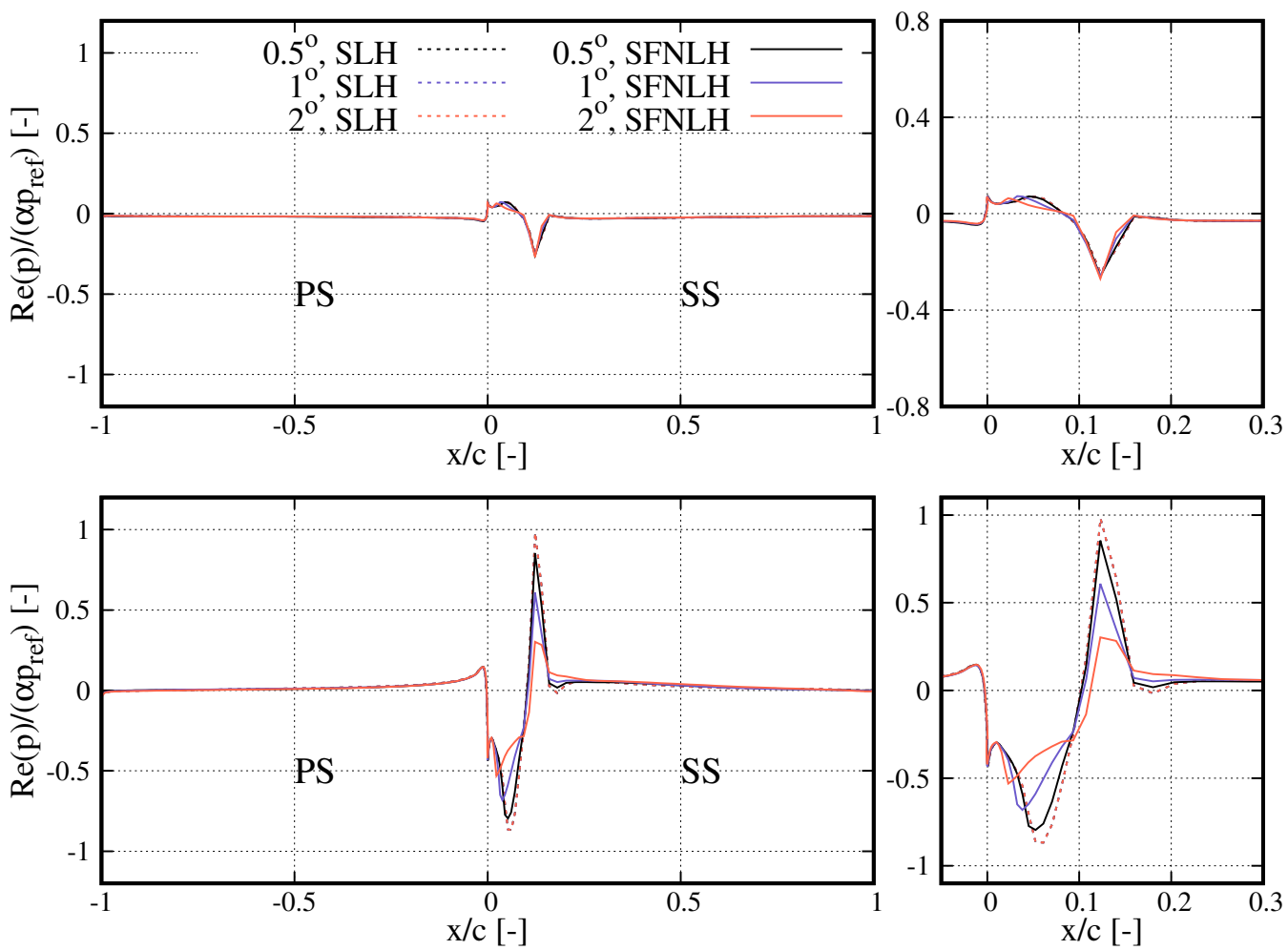

Fig. 10 Effect of deterministic fluxes on pressure variations with blade rotation.

\section{Velocity Variation}

The harmonic geometry distortion will also lead to mildly different velocity fields in each passage. The largest difference is observed in the axial velocity $u_{x}$. Similar to the previous comparisons, a Fourier transform of the direct whole assembly simulation is performed and it is compared with the flow perturbations computed by the proposed methods. Figure 11 shows the real and imaginary part of the velocity perturbations in the passage for the harmonic rotation of $1^{\circ}$ and their values are normalized by the larget $u_{x}$ variation in the passage. It can been that there are two regions where the largest perturbations are observed. The first one is around the transonic flow region on the suction side and the other one is in the wake region. Excellent agreement with the direct whole assembly solution is observed for both the real and imaginary parts .

The data in Fig. 11 is extracted on a pitch-wise line 5\% chord behind the cascade trailing edge and it is shown in Fig. 12. It can be seen from Fig. 11 that the value of the imaginary part is small, only the imaginary part is presented here. The variations of $u_{x}$ for the harmonic rotations of $0.5^{\circ}, 1^{\circ}$ and $2^{\circ}$ from SFNLH are compared with the direct whole assembly solutions. All the values are normalized by $u_{r e f}$ and $\alpha . u_{r e f}$ is the largest $\operatorname{Im}\left(u_{x}\right)$ when the rotation angle is $2^{\circ}$ and $\alpha$ is the corresponding rotation angle. In Fig. 11, pitch position $0 \%$ corresponds to the position on the lower periodic boundary and $100 \%$ corresponds to the upper periodic boundary. Good agreement with direct whole assembly simulations are observed for all cases and the non-linear response of the velocity field to the blade rotation is 


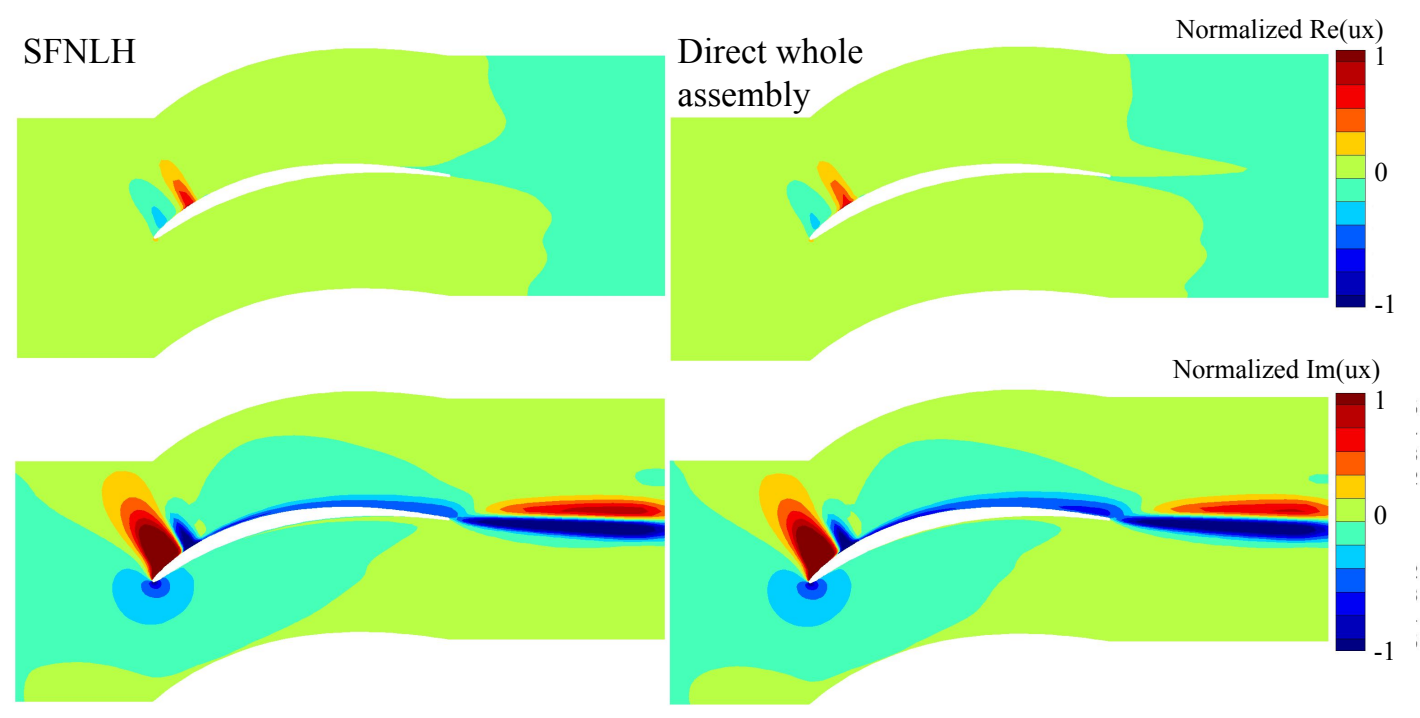

Fig. $11 u_{x}$ variations in the passage with blade rotation.

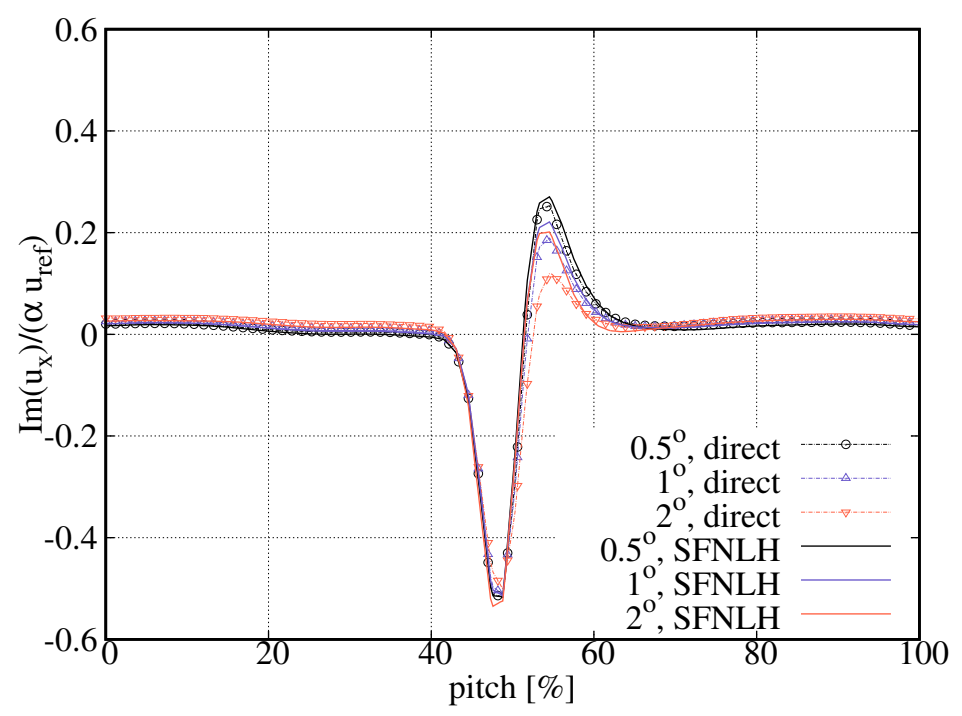

Fig. $12 u_{x}$ variations in the wake with blade rotation. 

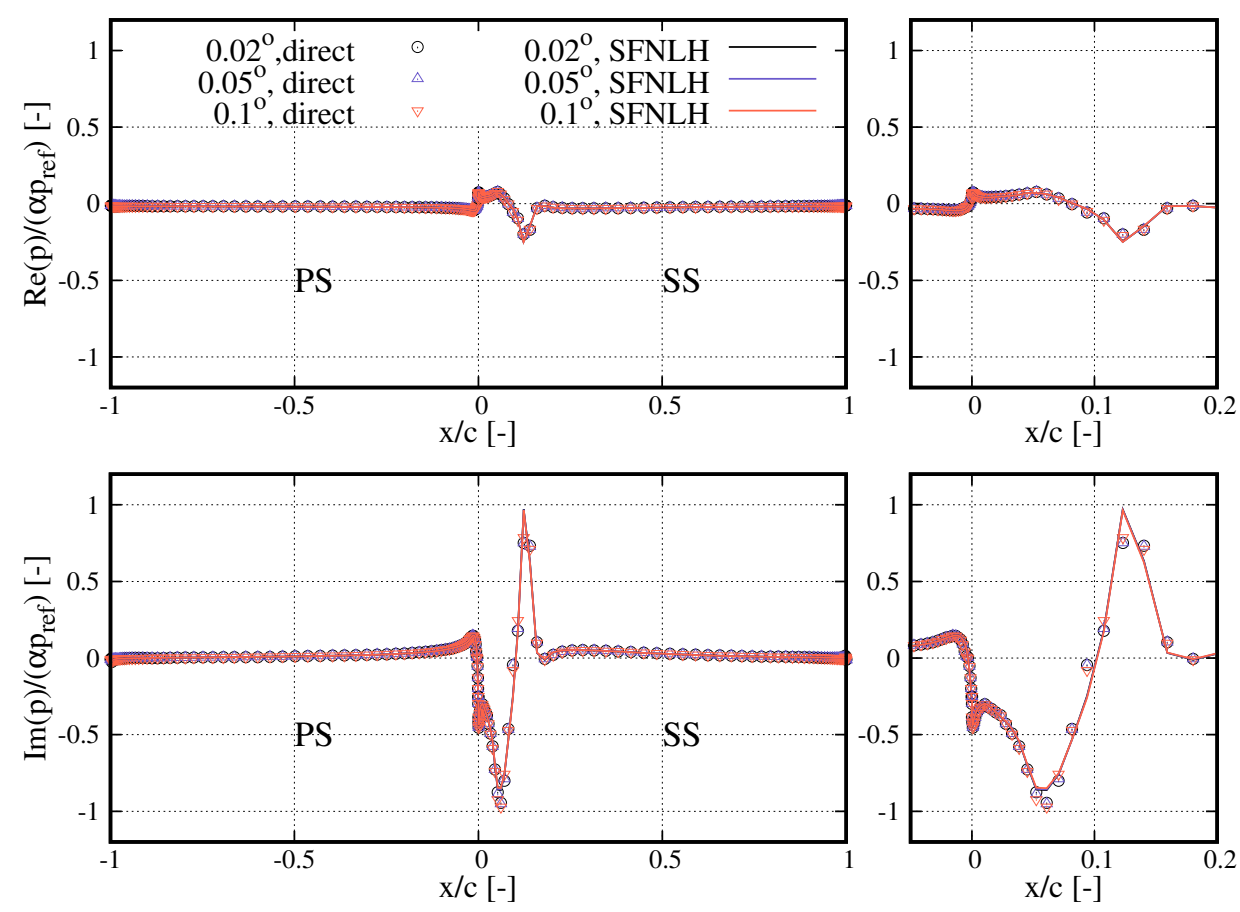

Fig. 13 Pressure variations on the blade with diminishing grid displacement.

clearly seen. As the rotation angle increases, there are slight over-prediction of $u_{x}$ perturbations around 55\% pitch position. This shows the boundary layer on the suction side exhibit a non-linear change due to blade rotation. It is also interesting to observe that the boundary layer on the pressure side shows much less non-linear effect. This is due to considerably less adverse pressure gradient on the pressure side compared to the suction side, as is shown in Fig. 6

\section{Asymptotic analysis with Diminishing Grid Displacement}

An asymptotic analysis is also conducted to demonstrate the correctness of the implementation of linearization (i.e. Equation 22 by allowing the rotation angles to approach zero. Besides, as the grid displacement is reduced towards zero, the non-linear effects, such as the ignored high order terms in Equation 13 and deterministic fluxes, are also diminishing. The solutions of SFNLH and the whole asembly solution should collapse into a single curve if a proper scaling factor is chosen. On the other hand, as all the non-linear effects are diminishing, the only approximation left is the frozen turbulence assumption. The discrepancies between SFNLH and the whole assembly solution can be a good assessment of the frozen turbulence assumption.

The blade is harmonically rotated by $0.02^{\circ}, 0.05^{\circ}$ and $0.1^{\circ}$. The resulting pressure variation on the blade is shown Fig. 13, and $u_{x}$ variations in the wake is shown Fig. 14. The pressures are normalized by $p_{r e f} \times \alpha$ and the velocity variation is normalized by $u_{r e f} \times \alpha . p_{r e f}$ is the largest $\operatorname{Im}(p)$ on the blade with a rotation angle of $0.1^{\circ}, u_{r e f}$ is the largest $\operatorname{Im}\left(u_{x}\right)$ when the rotation angle is $0.1^{\circ}$ and $\alpha$ is the corresponding rotation angle.

As is shown in Fig. 13 and Fig. 14, SFNLH solutions have a good agreement with the whole assembly solutions. 


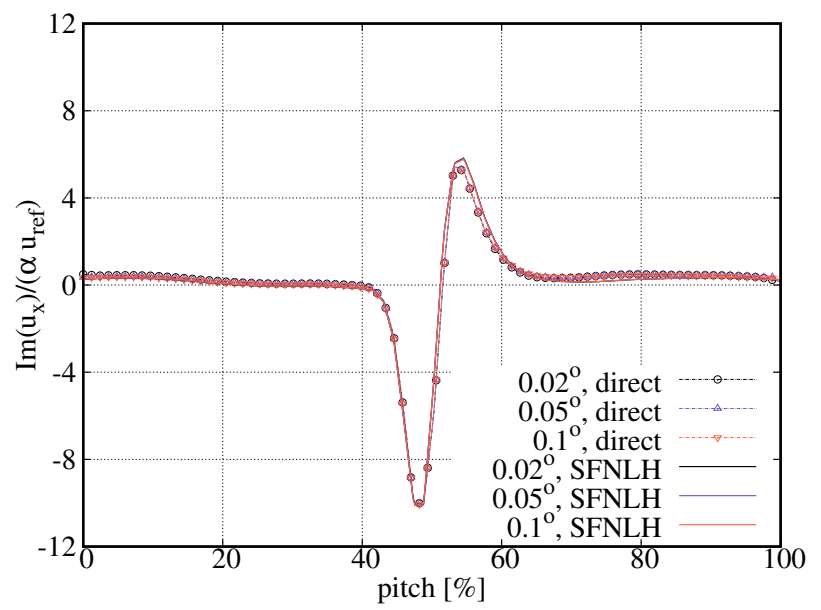

Fig. $14 u_{x}$ variations in the wake with diminishing grid displacement.

This demonstrates the correctness of the linearization. It can also be seen that for pressure variations, there are minor discrepancies on $5 \%$ to $15 \%$ chord of the suction side, where the flow is transonic. For the velocity variation in the wake, there are discrepencies around 55\% pitch position. These discrepancies are due to the modelling error introduced by the frozen turbulence assumption. However, it can be seen that such modelling error is of secondary importance and confined to a local region. Therefore frozen turbulence can be considered as a reasonably accurate approximation in the current work.

\section{Re-cambering}

The second case is the harmonic re-cambering of the assembly. Since the aerodynamic performance of a blade is sensitive to its camber [28], mild distortions are expected to achieve the design intent but minimize potential negative effect on the performance. To demonstrate the capability of the proposed method on handling re-cambering, the cascade is re-cambered in the way shown in Fig. 15. The geometry of a cascade can be represented in a local coordinate system $(\varepsilon, \eta)$. The blue dashed line in Fig. 15 represents the amount of displacement applied to the camber line and in this case the maximum re-cambering is at the mid-chord. After the new camber line is computed, the new blade is generated by applying the old thickness distribution on the new camber line. In Fig. 15, $T_{\max }$ is the maximum blade thickness, $\beta$ is the amount of re-cambering, which is taken as a percentage of $T_{\max }$, and it changes with one harmonic in the assembly with a wavelength of $2 \pi$, as is illustrated in Fig. 3

\section{Pressure Variation}

Three sets of computations are prepared for the blades which are re-cambered by $\beta=2.5 \%, 5 \%$ and $10 \%$ respectively. Figure 16 shows the pressure perturbations on the blade for SFNLH and whole assembly solutions. All the values are normalized by $p_{r e f}$ and $\beta . p_{\text {ref }}$ is the largest $\operatorname{Im}(p)$ on the blade when the amount of re-cambering is $10 \%$ and $\beta$ is the 


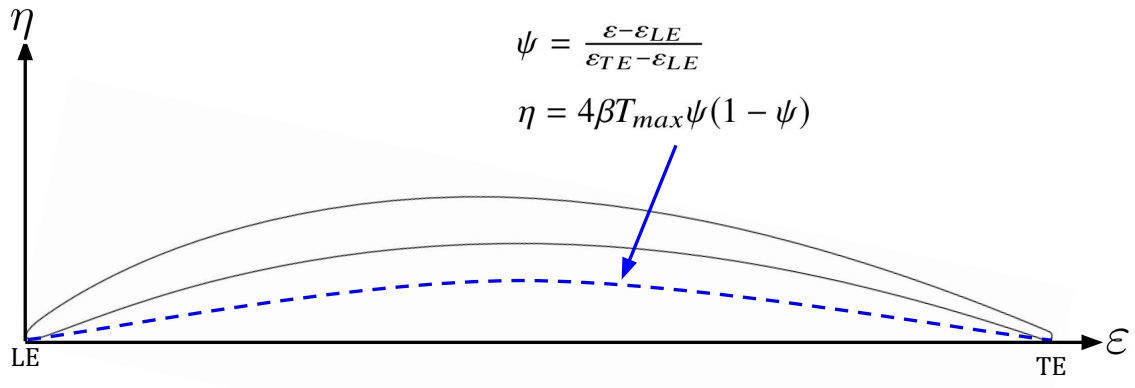

Fig. 15 Blade recambering.

corresponding re-cambering for each solution. As is mentioned before, such normalization will remove the linear effect and reveal the non-linear effects of the flow variations due to blade re-cambering.

Good agreement with the direct simulation is observed for all three cases. Similar to blade rotation, the largest pressure perturbation is observed on the suction side close to the leading edge, where the flow is transonic. Apart from this region, there are marginal pressure perturbations on the pressure side even the re-cambering is up to $10 \%$ of the blade thickness.

\section{Velocity Variation}

The variation of velocity is also examined and the distribution of the real and imaginary parts of axial velocity variations with $5 \%$ of blade thickness re-cambering is shown in Fig. 17 The values are normalized by the largest $\operatorname{Im}\left(u_{x}\right)$ in the domain. Good agreement with direct whole assembly simulation is observed. Besides similar patterns are found compared to the one shown in Fig. 11. There are large axial velocity variations in the transonic flow region on the suction side and in the wake region. $u_{x}$ perturbations in the wake region are also compared with the direct simulation and it is shown in Fig. 18 Since the real part is small, only the imaginary part is presented. The solutions from SLH is also added in the plot for comparisons. All the values are normalized by $u_{r e f}$ and $\beta$, $u_{r e f}$ is the largest $\operatorname{Im}\left(u_{x}\right)$ for a re-cambering of $10 \%$. Good agreement with the direct simulations is observed for all three re-cambering cases. Besides Fig. 18 shows similar trend as Fig. 12 and strong non-linear effects are observed around 55\% pitch position.

\section{E. Rotation and Re-cambering}

The flow perturbations due to single harmonic geometry perturbations (e.g. rotation or re-cambering) have been validated with the direct whole assembly solutions and good agreement have been observed between the proposed method and the direct solution for all the cases. In a real scenario, rotation and re-cambering are both present in the geometry distortions. To handle these combined geometry distortions, we can combine the flow perturbations due to their corresponding grid displacement linearly.

For example, to obtain the flow perturbations for $2^{\circ}$ rotation and $10 \%$ re-cambering, we could simply linearly 

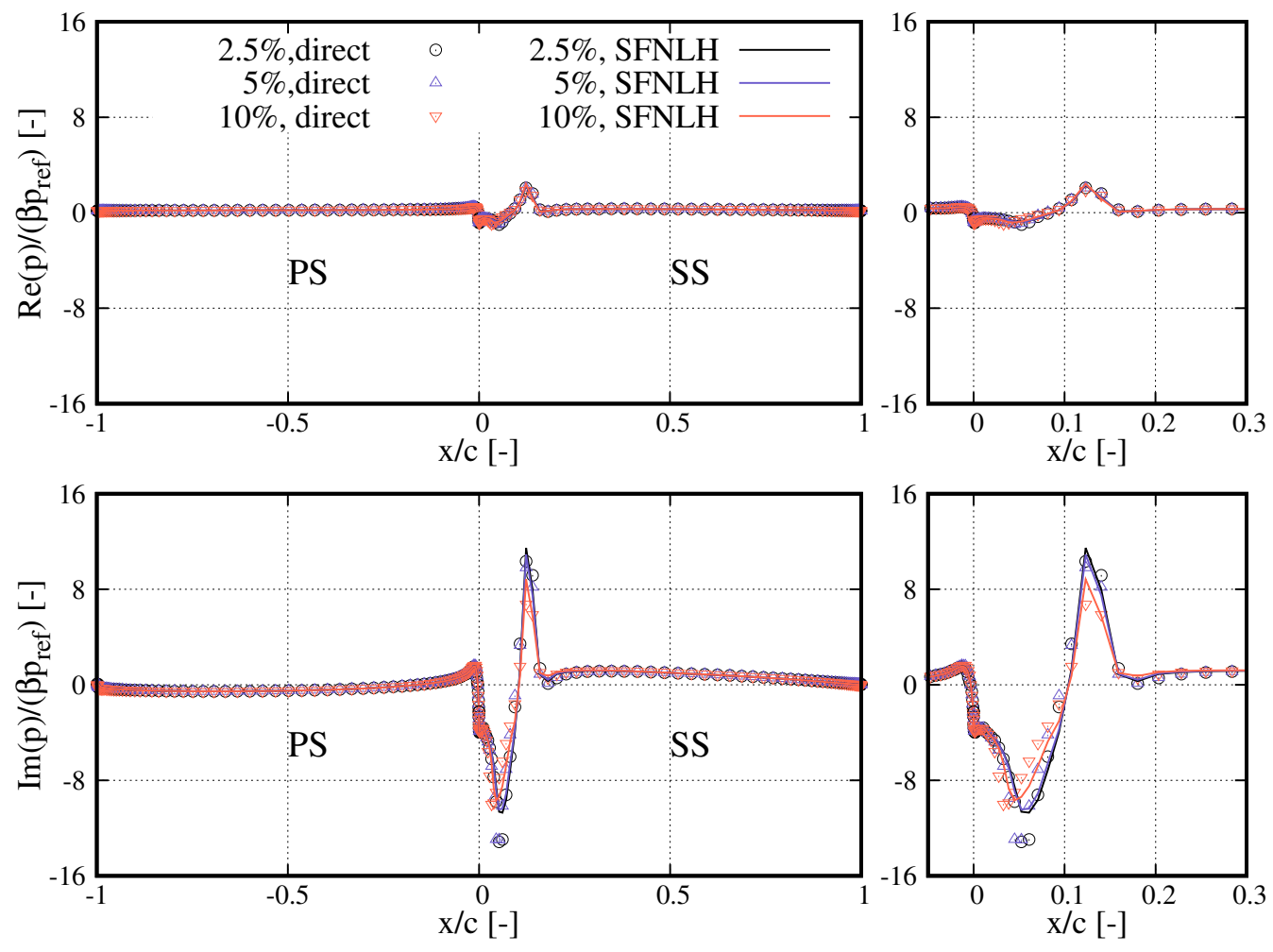

Fig. 16 Pressure variations on the blade with re-cambering.

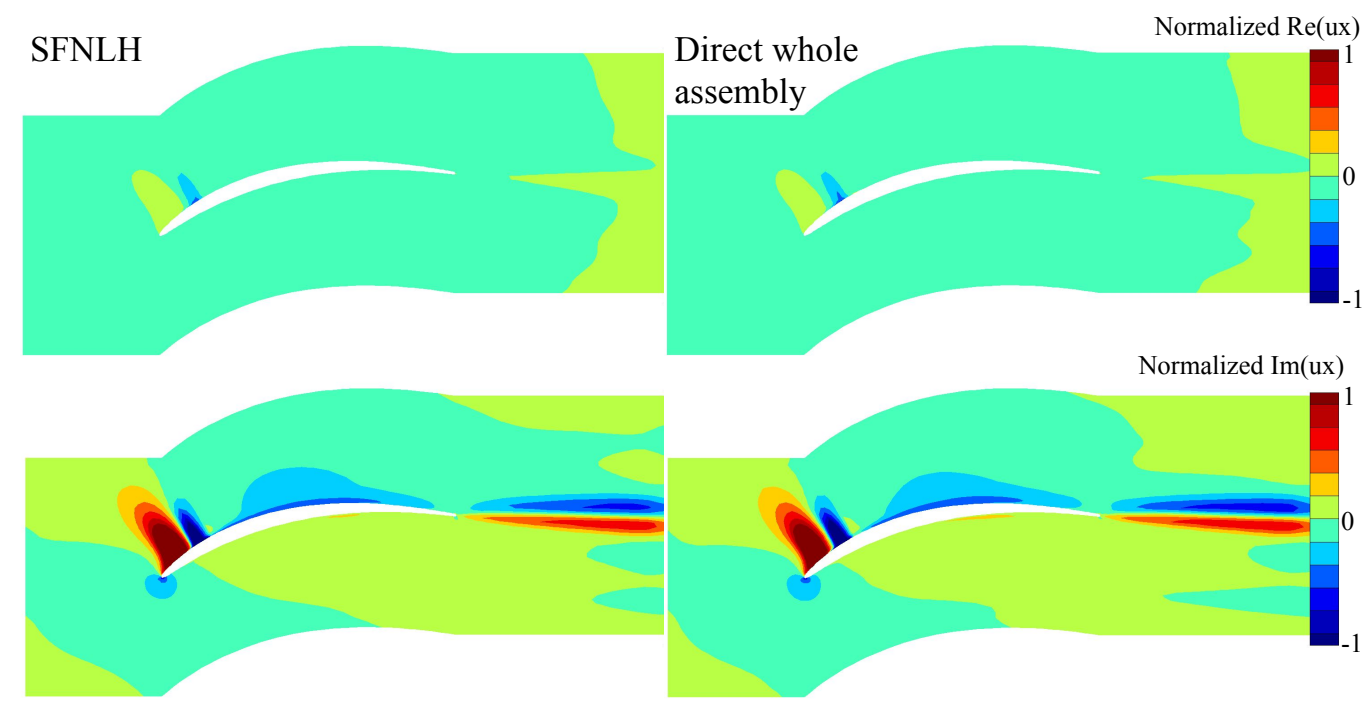

Fig. $17 u_{x}$ variations in the passage with re-cambering . 


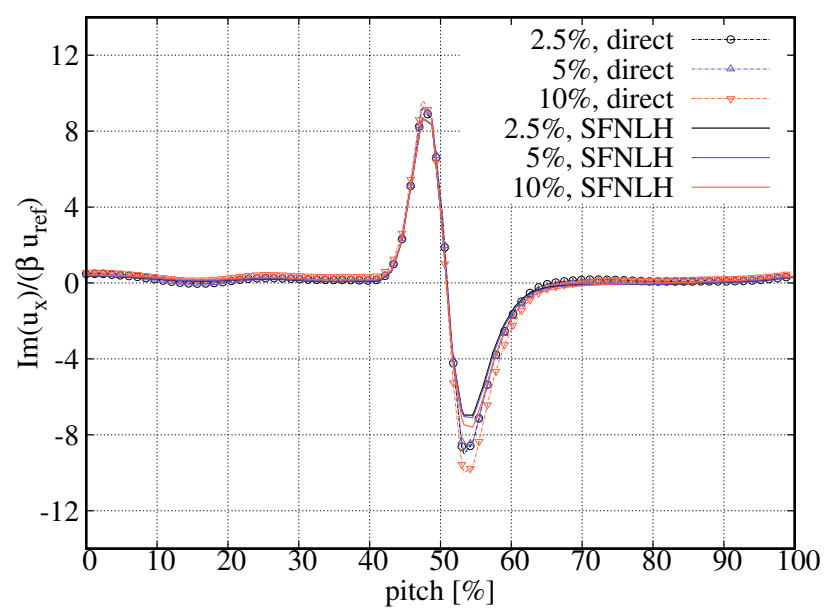

Fig. $18 u_{x}$ variations in the wake with re-cambering .

Table 1 Test matrix for combined rotation and recambering

\begin{tabular}{cccc}
\hline \hline & $10 \%$ recambering & $5 \%$ recambering & $2.5 \%$ recambering \\
\hline $2{ }^{\circ}$ rotation & $\checkmark$ & \\
$0.5^{\circ}$ rotation & $\checkmark$ & $\checkmark$ \\
\hline \hline
\end{tabular}

combine the single harmonic SFNLH solutions for $2^{\circ}$ rotation and $10 \%$ re-cambering. Therefore, for each harmonic solution, non-linear coupling between flow perturbations and the passage averaged flow is considered, but these solutions are linearly combined to produce flow perturbations for the final combined geometry distortions. The non-linear effects (e.g. the coupling of different harmonics) are lost but this strategy requires no further computations, once all the single harmonic solutions are computed. As will be shown, credible results can be obtained by such linear combinations compared to direct whole assembly solution with considerably less computational cost. To demonstrate the feasibility of this strategy, a test matrix shown in Table 1 for a set of combinations of rotation and re-cambering have been prepared.

From the single harmonic solution, it is shown previously that the real part is considerably small compared to the imaginary part, therefore, only the imaginary parts of the solutions are shown. Figure 19 shows the imaginary part of the pressure perturbations on the blade. The plot on the top left shows the comparisons with largest geometry distortion and the one on the bottom right shows the one with the smallest geometry distortion. All the values in the plots are normalized by the largest value of the imaginary part of the pressure perturbations with $2^{\circ}$ rotation and $10 \%$ recambering from the direct whole assembly solution. Remarkably, good agreement between the linear combinations and the direct solutions are observed for all cases. When geometry distortions become larger, there is increasing over-prediction of the pressure perturbations in the regions where the flow is transonic, but this region is confined to a small portion of the chord and in the reminder of the blade, the pressure perturbations are predicted accurately with the linear combinations.

The potential pressure field in front of the leading edge is also assessed. The data is extracted on a pitch-wise line sitting midway between the inlet and the leading edge. The results are shown in Fig. 20, Only the imaginary part is 




$0.5^{\circ}$ rotation $+10 \%$ recambering $\quad 0.5^{\circ}$ rotation $+5 \%$ recambering $\quad 0.5^{\circ}$ rotation $+2.5 \%$ recambering



Fig. 19 Linear combination of pressure variations on the blade.

shown. All the values in the plots are normalized by the largest value of the imaginary part of the pressure perturbations with $2^{\circ}$ rotation and $10 \%$ re-cambering from the direct solution. Excellent agreement is observed for all cases. It is interesting to notice that rotation leads to larger pressure perturbations compared to re-cambering, because it leads to larger loading variations in the assembly. This trend is captured well by the approach which uses the linear combination of the harmonic solutions.

The benefit of the linear combination of harmonic solutions can be envisaged as the following: suppose a designer would like to try $N_{r}$ harmonics for rotation and $N_{c}$ for re-cambering in the assembly. The linear combination strategy will only require $N_{r}+N_{c}$ single harmonic SFNLH solutions, whose computational cost is one order of magnitude less than the corresponding direct whole assembly one, and the linear combinations of these harmonic solutions, whose computational cost is negligible. In contrast, this will need $N_{c} \times N_{r}$ direct whole assembly solutions. As $N_{c}$ and $N_{r}$ become larger, such linear combination approach can lead to a reduction of the computational cost by several orders of magnitude compared to direct whole assembly simulations.

\section{Conclusion}

This paper has presented a method to efficiently compute aperiodic flows due to geometry distortions in an assembly. The capability of the method has been demonstrated in an OGV assembly with harmonic re-staggering and re-cambering. 


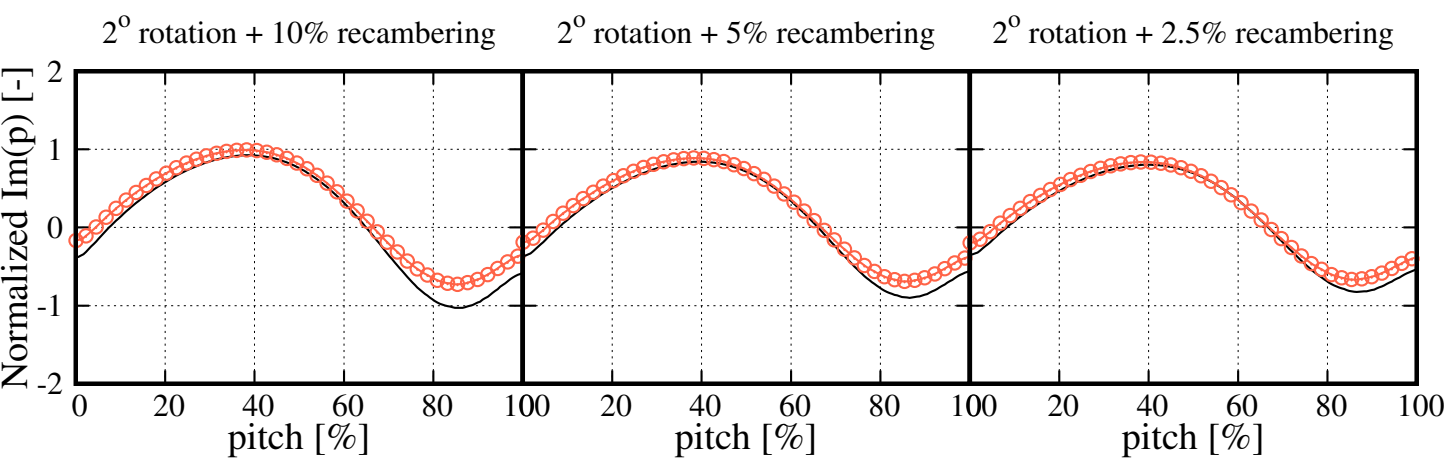

$0.5^{\circ}$ rotation $+10 \%$ recambering $\quad 0.5^{\circ}$ rotation $+5 \%$ recambering $\quad 0.5^{\circ}$ rotation $+2.5 \%$ recambering

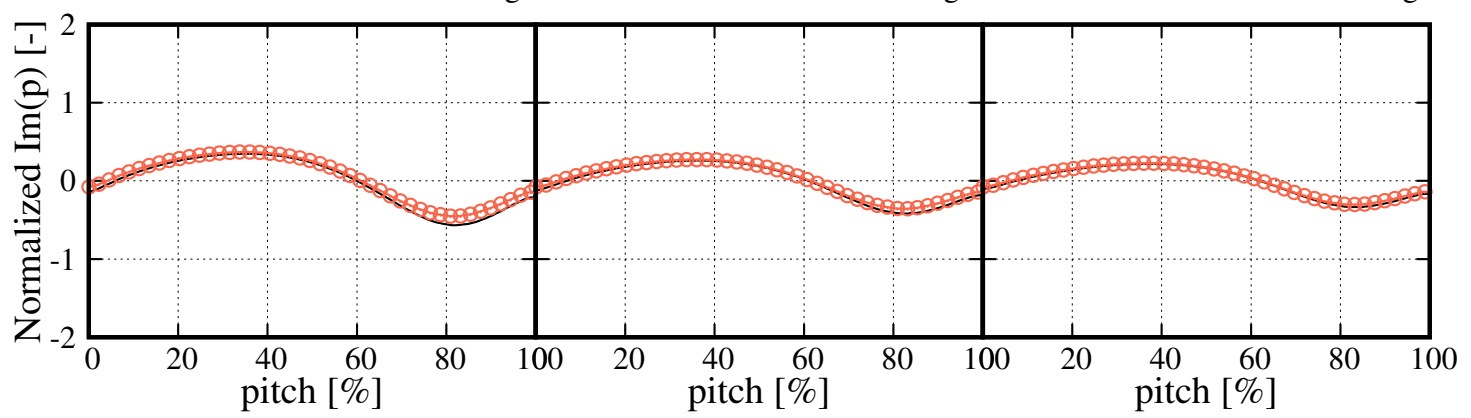

Linear combination of Fourier solution

Direct whole assembly

Fig. 20 Linear combination of pressure variations at inlet.

Good agreement on the flow fields between the proposed method and the direct whole assembly simulations are observed on all cases. The effect of deterministic fluxes are observed on the transonic flow region on the suction side. If no deterministic fluxes are considered, the pressure fluctuation in the transonic flow region are over-estimated.

The non-linear/linear effects of the flow perturbations to geometry distortions is also examined. It is demonstrated that the response of pressure perturbations on geometry distortion is largely linear apart from the transonic flow region on the suction side. Stronger non-linear effects are observed in the velocity fields. The discrepancies between SFNLH and the whole assembly solution increase with larger geometry modifications.

The harmonic solutions due to re-staggering and re-cambering are then linearly combined to demonstrate the feasibility of the approach in a design enviroment. The resulting pressure perturbations in the assembly are compared with direct whole assembly solutions. Good agreement is observed on the pressure perturbations on the blade and the potential pressure field in front of the blade. There is slight over-prediction of the pressure perturbation on a small portion (roughly $2 \%$ chord length) on the blade suction side. However, considering that the computational cost of the linear combination of harmonic solutions can be several order magnitude cheaper than the direct whole assembly solutions and the integral of the unsteady pressure is of primary interest, this approach can be a useful tool at the design stage where timely results are required to examine a large volume of designs.

Finally, although the non-linear coupling between the flow perturbations in the assembly and the passage-averaged 
mean flow is considered, it is noted that the mathematical formulation to connect grid displacement and flow perturbation is still based on linear theory. Therefore the non-linear effects between grid displacement and flow perturbations are not included. In all the test cases with mild geometry distortions, such effect is found to be of secondary importance but become increasingly noticeable in the cases with relatively large geometry modifications (e.g. $2^{\circ}$ or rotation and $10 \%$ of re-cambering). This can be seen from the over-prediction of velocity perturbations in the wake as is shown Fig. 12 and Fig. 18 Future work is to address this issue.

\section{Acknowledgments}

The authors would like to thank Rolls-Royce plc. for funding this work and granting the permission for publication. The authors would also like to thank John Adamczyk for discussion and his comments on this paper.

\section{References}

[1] Barber, T. J., and Weingold, H. D., "Vibratory Forcing Functions Produced by Nonuniform Cascades,” Journal of Engineering for Power, Vol. 100, No. 1, 1978, p. 82. doi:10.1115/1.3446330, URL https://doi.org/10.1115/1.3446330

[2] Kodama, H., "Performance of Axial Compressor With Nonuniform Exit Static Pressure," Journal of Turbomachinery, Vol. 108, No. 1, 1986, p. 76. doi:10.1115/1.3262027, URL https://doi.org/10.1115/1.3262027.

[3] Kodama, H., and Nagano, S., "Potential Pressure Field by Stator/Downstream Strut Interaction,” Journal of Turbomachinery, Vol. 111, No. 2, 1989, p. 197. doi:10.1115/1.3262256, URL https://doi.org/10.1115/1.3262256

[4] Rife, M. E., Barbarossa, F., di Mare, L., Parry, A. B., and Green, J. S., "Radical Guide Vane Design and Optimization,” Journal of Propulsion and Power, Vol. 34, No. 6, 2018, pp. 1455-1466. doi:10.2514/1.b36663, URL https://doi.org/10.2514/1. b36663.

[5] He, L., and Ning, W., "Efficient Approach for Analysis of Unsteady Viscous Flows in Turbomachines," AIAA Journal, Vol. 36, No. 11, 1998, pp. 2005-2012. doi:10.2514/2.328, URL https://doi.org/10.2514/2.328

[6] Hall, K. C., Thomas, J. P., and Clark, W. S., "Computation of Unsteady Nonlinear Flows in Cascades Using a Harmonic Balance Technique," AIAA Journal, Vol. 40, No. 5, 2002, pp. 879-886. doi:10.2514/2.1754, URL https://doi.org/10.2514/2. 1754

[7] Sicot, F., Puigt, G., and Montagnac, M., "Block-Jacobi Implicit Algorithms for the Time Spectral Method," AIAA Journal, Vol. 46, No. 12, 2008, pp. 3080-3089. doi:10.2514/1.36792, URL https://doi.org/10.2514/1.36792

[8] Ekici, K., and Hall, K. C., "Nonlinear Analysis of Unsteady Flows in Multistage Turbomachines Using Harmonic Balance," AIAA Journal, Vol. 45, No. 5, 2007, pp. 1047-1057. doi:10.2514/1.22888, URL https://doi .org/10.2514/1.22888 
[9] Hall, K. C., Ekici, K., Thomas, J. P., and Dowell, E. H., "Harmonic balance methods applied to computational fluid dynamics problems," International Journal of Computational Fluid Dynamics, Vol. 27, No. 2, 2013, pp. 52-67. doi: 10.1080/10618562.2012.742512, URL https://doi.org/10.1080/10618562.2012.742512

[10] Vilmin, S., Lorrain, É., Tartinville, B., Capron, A., and Hirsch, C., "The nonlinear harmonic method: from single stage to multi-row effects," International Journal of Computational Fluid Dynamics, Vol. 27, No. 2, 2013, pp. 88-99. doi: 10.1080/10618562.2012.752074, URL https://doi.org/10.1080/10618562.2012.752074

[11] McMullen, M., and Jameson, A., "The computational efficiency of non-linear frequency domain methods," Journal of Computational Physics, Vol. 212, No. 2, 2006, pp. 637-661. doi:10.1016/j.jcp.2005.07.021, URL https://doi.org/10. $1016 / j \cdot j c p .2005 .07 .021$

[12] Wang, F., and di Mare, L., "Favre-Averaged Nonlinear Harmonic Method for Compressible Periodic Flows," AIAA Journal, Vol. 57, No. 3, 2019, pp. 1133-1142. doi:10.2514/1.j057523, URL/https://doi.org/10.2514/1.j057523

[13] He, L., "Fourier Modeling of Steady and Unsteady Nonaxisymmetrical Flows," Journal of Propulsion and Power, Vol. 22, No. 1, 2006, pp. 197-201. doi:10.2514/1.15701, URL https://doi.org/10.2514/1.15701

[14] Adamczyk, J. J., “Aerodynamic Analysis of Multistage Turbomachinery Flows in Support of Aerodynamic Design,” Journal of Turbomachinery, Vol. 122, No. 2, 2000, p. 189. doi:10.1115/1.555439, URL https://doi .org/10.1115/1.555439.

[15] Wilcox, D. C., Turbulence Modeling for CFD (Third Edition), D C W Industries, 2006. URL https://www . amazon. com/ Turbulence-Modeling-Third-David-Wilcox/dp/1928729088?SubscriptionId=0JYN1NVW651KCA56C102\&tag= techkie $-20 \&$ linkCode $=x m 2 \&$ camp $=2025 \&$ creative $=165953 \&$ creativeASIN $=1928729088$

[16] Cebeci, T., Turbulence Models and Their Application: Efficient Numerical Methods with Computer Programs, Springer, 2004. URL https://wWw.amazon.com/Turbulence-Models-Their-Application-Efficient/dp/3540402888? SubscriptionId=0JYN1NVW651KCA56C102\&tag=techkie-20\&linkCode=xm2\&camp=2025\&creative=165953\& creativeASIN $=3540402888$

[17] Roe, P., "Approximate Riemann solvers, parameter vectors, and difference schemes," Journal of Computational Physics, Vol. 43, No. 2, 1981, pp. 357-372. doi:10.1016/0021-9991(81)90128-5, URL https://doi .org/10.1016/0021-9991(81)901285

[18] di Mare, L., Kulkarni, D. Y., Wang, F., Romanov, A., Ramar, P. R., and Zachariadis, Z. I., "Virtual Gas Turbines: Geometry and Conceptual Description,” Proceedings of ASME TurboExpo, Vancouver, Canada, ASME, 2011. doi:10.1115/gt2011-46437, URL https://doi.org/10.1115/gt2011-46437

[19] Wang, F., Carnevale, M., Lu, G., di Mare, L., and Kulkarni, D., "Virtual Gas Turbine: Pre-Processing and Numerical Simulations," Proceedings of ASME TurboExpo, South Korea, Seoul, ASME, 2016. doi:10.1115/gt2016-56227, URL https://doi.org/10.1115/gt2016-56227 
[20] Mavriplis, D., "Revisiting the Least-Squares Procedure for Gradient Reconstruction on Unstructured Meshes," 16th AIAA Computational Fluid Dynamics Conference, American Institute of Aeronautics and Astronautics, 2003. doi:10.2514/6.2003-3986, URL https://doi.org/10.2514/6.2003-3986.

[21] Hadade, I., Wang, F., Carnevale, M., and di Mare, L., "Some useful optimisations for unstructured computational fluid dynamics codes on multicore and manycore architectures," Computer Physics Communications, Vol. 235, 2019, pp. 305-323. doi:10.1016/j.cpc.2018.07.001, URL https://doi.org/10.1016/j.cpc.2018.07.001

[22] Luo, H., Baum, J. D., and Löhner, R., “A Fast, Matrix-free Implicit Method for Compressible Flows on Unstructured Grids," Journal of Computational Physics, Vol. 146, No. 2, 1998, pp. 664-690. doi:10.1006/jcph.1998.6076, URL https: //doi.org/10.1006/jcph.1998.6076

[23] Blazek, J., Computational fluid dynamics : principles and applications, $2^{\text {nd }}$ ed., Butterworth Heinemann, Oxford, UK, 2015. Page 184-185.

[24] F. R. Menter, M. K., and Langtry, R., “Ten Years of Industrial Experience with the SST Turbulence Model,” Proceedings of the 4th International Symposium on Turbulence, Heat and Mass Transfer, Begell House Inc., West Redding, USA, 2003, pp. 625-632.

[25] Carnevale, M., Wang, F., and di Mare, L., “Calculation of Intake-Fan-Bypass Interaction With a Fan Similarity Model,” Volume 2C: Turbomachinery, ASME, 2018. doi:10.1115/gt2018-75115, URL https://doi .org/10.1115/gt2018-75115.

[26] Wang, F., "Whole aero-engine meshing and CFD simulation," Ph.D. thesis, Imperial College London, Nov 2013. Http://hdl.handle.net/10044/1/27235.

[27] Wang, F., and di Mare, L., "Mesh Generation for Turbomachinery Blade Passages with Three-Dimensional Endwall Features," Journal of Propulsion and Power, Vol. 33, No. 6, 2017, pp. 1459-1472. doi:10.2514/1.b36356, URL https: //doi.org/10.2514/1.b36356

[28] Cumpsty, N., Compressor Aerodynamics, $2^{\text {nd }}$ ed., Krieger Publishing, Floria, USA, 2004. 